\title{
Competitividad y especialización en el Bajío mexicano: ¿acaso una región homogénea?
}

\section{Kurt Unger*, Diana Flores** y Lizet A. Pérez $* * *$}

\section{RESUMEN}

Se estima la competitividad y especialización sectorial de la Región Bajío. En general, el Bajío padece de poca competitividad, tanto por baja productividad como por nivel de salarios. No obstante, las localidades del Bajío son muy heterogéneas en competitividad. Tres localidades alcanzan condición competitiva: Querétaro, Guadalajara e Irapuato. Las otras 18 Zonas Metropolitanas (ZM) y municipios del Bajío califican muy por debajo. La media docena de ciudades o ZM que integran el grupo o cluster 1 son significativamente más competitivas que los otros. El resto tienen una competitividad sistémica de alcances muy modestos, sean municipios medianos y también los otros municipios de menor tamaño. En general, registran muy poca actividad competitiva y la mayoría se caracterizan como monoproductores clásicos que no generan derramas de significancia.

Palabras clave: Especialización, Competitividad, Regiones.

Clasificación JEL: R12, D24, J24.

* Profesor investigador de la División de Economía, Centro de Investigación y Docencia Económicas, A.C., México. Correo electrónico: kurt.unger@cide.edu. Una versión preliminar se publicó en la serie Documentos de Trabajo CIDE E-569, 2014. Agradezco la colaboración de Luis Fueyo y Geraldine Mendoza en la preparación de esta versión.

** Asistente de Investigación en 2012 y egresada de la Licenciatura en Economía de la Universidad Autónoma de Aguascalientes, México. Correo electrónico: diana.floresperegrina@gmail. com

*** Asistente de Investigación en 2013 y egresada de la Licenciatura en Economía del Centro de Investigación y Docencia Económicas, A.C., México. Correo electrónico: alejandrapc1503@ gmail.com 


\section{ABStract}

\section{Competitivity and Specialization in the Bajio Region of Mexico: Is}

\section{it An Homogeneous Region?}

We estimate the competitiveness of the Región Bajío. The overall assessment is that Bajío lacks competitiveness, lagging in both labor productivity and wage levels. However, there are quite heterogeneous competitive conditions. Only three localities reach a favorable level of competitiveness: Querétaro, Guadalajara and Irapuato. The other 18 metropolitan zones (ZM) and municipalities show very low levels of competitiveness. Applying cluster analysis there are half a dozen cities with higher and spread competitive conditions. The rest show a limited systemic integration, including small municipalities mostly characterized as monoproducers that have not created significant spillovers to other activities.

Keywords: Specialization, Competitiveness, Regions.

JEL Classification: R12, D24, J24.

\section{INTRODUCCIÓN}

En este trabajo se busca extender la identificación de las características de la competitividad económica de los municipios y Zonas Metropolitanas (ZM) principales que integran la región Bajío, a fin de definir las ventajas reveladas de cada uno. El interés ahora se centra en estimar las condiciones competitivas de los municipios y las ZM importantes de los siete estados que, en principio, guardan cierta relación expresada en la red carretera sobre la que versó un ejercicio anterior (Unger et al., 2014a, ver anexo 1). El análisis se hace considerando la trayectoria productiva y competitiva de las principales actividades de cada ZM y municipio, su relación con la dotación disponible de recursos naturales locales y con las potencialidades que ofrecen para atraer a las nuevas inversiones. Más allá de algunos rasgos comunes entre ellos, sobresalen especializaciones competitivas muy puntuales a cada ZM y municipio, observándose más diferencias que semejanzas en la mayoría de los casos. El resultado pretende orientar la especialización del desarrollo futuro a partir de acciones de política regional más específicas a cada situación.

Otros trabajos previos (Unger, 2012) han mostrado la conformación de regiones en México con distintos grados de industrialización y 
especialización productiva. En este caso se hace a través de identificar las fuentes de las ventajas competitivas que se observan para dichas actividades en las distintas ZM y localidades de la Región Bajío. El resultado esperado es comprobar que existen diferencias sustantivas en el grado de industrialización e integración entre actividades en prácticamente todos los niveles geográficos, como reflejo de la importancia que imponen algunas actividades al entorno de ciertas localidades específicas. Partimos de distinguir actividades de dos tipos. Las que se desarrollan bajo el liderazgo de productos y servicios regionalmente muy especializados y concentrados en pocas localidades. Estas son por su propia naturaleza actividades competitivas de origen comerciable, y que dominan la dinámica local a partir de competir en el ámbito de la globalización de los mercados. El otro tipo de actividades comprenden la mayoría de los servicios y comercios que tienden a desarrollarse cerca de cada mercado y en atención a la dinámica de la población en condición de seguidores de las primeras. Estas actividades generalmente son no comerciables, ya que disfrutan de condiciones de protección natural, y su desempeño puede depender en mayor grado de la dinámica del primer grupo. Hacia el final del análisis se verá que la competitividad de estas actividades no comerciables es más similar entre diversas localidades.

Si bien se distinguen las condiciones de mercado de inicio, también se intenta destacar la integración de actividades cercanamente relacionadas en clusters sectoriales que pueden explicar en mayor grado las ventajas de crecimiento que manifiestan unas zonas geográficas por sobre otras.

En el ámbito mexicano se acostumbra trabajar en regiones agrupadas que suman varios estados por vecindad geográfica y por similitud de condiciones en lo económico, en lo demográfico y por territorialidad (véase Corral, 2000). La evidencia acerca de actividades económicas y concentraciones de población en el país que desbordan los límites político administrativos de origen de municipios y estados, han dado paso a la delimitación oficial de un número considerable de Zonas Metropolitanas (Sedesol, Conapo, Inegi 2004). El Bajío puede entonces considerarse, de principio, como una misma región desde ciertos criterios de análisis, aunque el que aquí se presenta subrayará diferencias entre las ZM principales dentro de la Región. 
En México la convergencia también se ha cuestionado. En el contexto mexicano y del Bajío, hay algunos antecedentes destacados como el de Abdel (2000) con su estudio sobre el tejido empresarial en Aguascalientes, Estrada (2006) en referencia a los recursos de ciencia, tecnología e innovación en Guanajuato, y la OECD (2009) con su reporte de clusters de innovación en 15 estados mexicanos, incluyendo los de la zona Bajío (Aguascalientes, Guanajuato, San Luis Potosí, Querétaro, Jalisco, Michoacán y Zacatecas).

Otros antecedentes en la OECD (2007) van en una dirección similar al destacar las características técnico-industriales en los estudios sobre clusters de innovación. En todos los casos, el desempeño observado no puede separarse de la base técnica-industrial o comercial heredada en la región en que se desarrollan las redes que integran a cada cluster (Rodríguez-Posé, 2000). Este enfoque industrial-sectorial-nodal es el que aquí también se privilegia.

El trabajo contiene cuatro secciones. En la primera definimos la Región Bajío a nivel municipios. La segunda identifica las actividades productivas de mayor relevancia y su concentración en los municipios. La tercera introduce dos aspectos para medir la competitividad y, finalmente, la sección de conclusiones.

\section{ZONAS METROPOLITANAS Y MUNICIPIOS IMPORTANTES EN EL BAJío}

\subsection{Introducción a la región}

El propósito es caracterizar la economía y competitividad económica del Bajío, considerando aspectos tales como la importancia del producto interno bruto, la productividad, el empleo, los salarios y otras características de la organización industrial y tecnológica de las actividades destacadas en las Zonas Metropolitanas del Bajío. Esto nos permitirá situar el origen de la competitividad en los diversos sectores de manufactura, comercio, servicios, minería, infraestructura, construcción y actividades agro-industriales a efectos de apreciar el grado de congruencia entre las ventajas competitivas sectoriales "reveladas" en la Región, con las prioridades de política para impulsar las vocaciones regionales y locales. Es obvio que no todas las Zonas Metropolitanas y municipios han alcanzado las mismas condiciones de desarrollo actual, 
y posiblemente tampoco tienen las mismas expectativas a futuro dada la influencia que predomina en forma de tendencia inercial.

Como se argumentó en Unger et al. (2014b), la hipótesis de principio es que aunque las manufacturas siguen presentándose con los mayores rendimientos de especialización virtuosa en general, las ventajas "reveladas" parecen encontrarse en muy diversas circunstancias que es necesario precisar. En un sentido alternativo al argumento de industrialización virtuosa, también hay casos de actividades industriales maduras con una dinámica más moderada que conviene tener en cuenta para considerar los mecanismos y políticas más adecuados a su reconstrucción. Muchos de estos casos se relacionan con industrias tradicionales que extendieron demasiado el privilegio de protección al mercado interno o bien de actividades cuya orientación maquiladora determina condiciones competitivas que son, por su propia, naturaleza de evolución incierta.

La idea de las regiones como polos de competitividad ha llevado a la búsqueda de los factores que interactúan para dar lugar a las concentraciones de población y de la actividad económica. Las unidades básicas de esta dinámica de aglomeración son las ciudades, cuya aparición resulta de la tensión entre dos fuerzas que operan en sentido contrario (Fujita, Krugman y Venables, 1999). Por un lado, las economías de escala $^{1}$ que están asociadas con la proximidad geográfica y promueven la concentración (fuerzas centrípetas). Por otro lado, las deseconomías ${ }^{2}$ que son inherentes al tamaño de una ciudad e inhiben la concentración (fuerzas centrífugas). El resultado de estas interacciones, en el caso mexicano y en particular en el Bajío, es un conjunto de localidades de diversos tamaños, más o menos especializadas en algunas actividades económicas y que se encuentran distribuidas sobre el territorio. La conformación de ZM integradas por varios municipios que comparten una misma lógica de desarrollo ha pasado a ser más común que la inde-

1 Las economías de escala o externas (externalidades positivas) derivadas de la concentración espacial atrajeron la atención de Alfred Marshall desde 1920. Pueden ser de tres tipos: i) derramas tecnológicas y de conocimiento, ii) amplio mercado de trabajo especializado y iii) el desarrollo de los vínculos progresivos y regresivos inherentes a las concentraciones en grandes mercados locales.

2 En la literatura se ha hecho mención a diversos factores que desalientan la concentración (Krugman, 1991; Baldwin et al., 2003). Por ejemplo, un mayor grado de competencia, los costos derivados de la congestión, la sobrepoblación y la contaminación. 
pendencia relativa entre estos. En esos casos, las ZM se conforman con diversas ciudades y poblaciones aledañas, ofreciendo cada una ciertas partes del funcionamiento agregado, en muchos casos con zonas industriales separadas de las habitacionales (INEGI, 2012).

El acelerado crecimiento de este tipo de urbes se hizo más frecuente a partir de la segunda mitad del siglo XX. En México, muchas ciudades en su proceso expansivo suelen desbordar los límites político-administrativos de los municipios que originalmente las contenían, dando así origen a las $\mathrm{ZM}^{3}$, que en ocasiones trascienden también los límites estatales. El estudio del fenómeno metropolitano en México adquiere importancia durante la década de los setenta, periodo en el que surge el mayor número de ZM y además, el concepto es reconocido por el gobierno federal en el Sistema Nacional de Planeación del Desarrollo Urbano (Sobrino, 2003). Tomando en cuenta estos elementos, nos proponemos analizar la conformación de la Región Bajío.

\subsection{La configuración del Bajío: Zonas Metropolitanas y municipios de importancia}

Toda delimitación de regiones supone un cierto grado de discrecionalidad para fijar los límites. Una región debe de compartir al menos una característica que la haga homogénea en su interior y heterogénea al exterior (Meyer, 1963). Las regionalizaciones pueden responder a distintos criterios que van desde factores históricos, culturales, políticos, geográficos y económicos, hasta los naturales. La delimitación que nosotros proponemos de la Región Bajío fue en atención a dos criterios; primero el referente a la identificación geográfica de ciudades o puntos del territorio y el segundo referido a la importancia y a la dinámica económica de las localidades que integran las ZM y otros municipios cercanos.

La tendencia internacional es la misma. En 1950 había tan sólo ocho ciudades en el mundo con una población mayor a cinco millones de habitantes, para el año 2000 ya sumaban 41 (United Nations, 1999). En México, no es sino hasta 2004, cuando el gobierno federal adopta una definición operativa de ZM que reúne los criterios de tres instituciones - Inegi, Conapo y Sedesol - que anteriormente ofrecían una definición atendiendo a sus propios criterios de interés. Una ZM es el conjunto de dos o más municipios donde se localiza una ciudad de 50 mil o más habitantes, cuya área urbana, funciones y actividades rebasan el límite del municipio que originalmente la contenía, incorporando como parte de sí misma o de su área de influencia directa a municipios vecinos, predominantemente urbanos, con los que mantiene un alto grado de integración socioeconómica (Sedesol, Conapo, Inegi 2004). 
El primer criterio privilegia la unión de territorios, principalmente a partir de la red carretera. La delimitación que aquí proponemos encuentra sustento en el ejercicio de Chías et al. (2010), en que se analiza la influencia del transporte carretero en la organización del territorio mexicano. En el trabajo previo de Unger et al. (2014a) se definió la región Bajío atendiendo a este criterio. Inicialmente, se eligió la totalidad de siete estados que han sido considerados en distintos estudios del Bajío: Aguascalientes, Guanajuato, Jalisco, Michoacán, Querétaro, San Luis Potosí y Zacatecas. Posteriormente se delimitó el territorio a un conjunto de 210 municipios $^{4}$ alrededor del polígono que se forma siguiendo las carreteras federales - de cuota y libres - de la región. Una apreciación de conjunto puede verse en el mapa 1 de aquel ejercicio (incluído en el Anexo 1 al final de este documento), en el cual la región Bajío se comprende por las partes altas de los estados de Jalisco y Michoacán, la totalidad de los estados de Querétaro, Guanajuato y Aguascalientes, y las partes bajas de los estados de San Luis Potosí y Zacatecas.

El segundo criterio considera las localidades que destacan económicamente y se posicionan como líderes regionales, marcando la pauta en el desarrollo de la región en donde se encuentran. Desde esta perspectiva, las ZM representan nodos de crecimiento y desarrollo que pueden difundir los beneficios al resto del sistema (Corral, 2000). La aproximación de este criterio territorial se basa en la definición de ZM del Inegi, a saber, una ZM es todo conjunto de dos o más municipios donde se localiza una ciudad de 50 mil o más habitantes, cuya área urbana, funciones y actividades rebasan el límite del municipio que originalmente la contenía; y se extiende a todos aquellos municipios que contienen una ciudad de un millón o más habitantes. ${ }^{5}$

Con base en este criterio, la elección más puntual de las unidades de análisis considera la definición de Zonas Metropolitanas del INEGI y la

4 Estos municipios son casi la mitad de los 429 municipios que conforman los siete estados originales. El resto de los municipios (209), es decir, los que quedan fuera del polígono carretero, no son considerados en nuestro ejercicio, pues quedan muy lejos de la comunicación por carretera y de la dinámica económica que se da entre los integrantes del polígono.

5 El Inegi considera otros criterios más específicos para definir diferencias entre municipios: Municipios centrales, municipios exteriores por criterios estadísticos y geográficos y municipios exteriores por planeación y política urbana (ver Inegi, 2012). 
importancia económica de la unidad, esto es, que cada ZM o municipio aporte individualmente más del $0.5 \%$ del valor agregado (VA) de la región $^{6}$. De tal forma, se integran 31 municipios en $10 \mathrm{ZM}$ y se añaden a éstas 15 municipios atendiendo al criterio de importancia económica. Estos se componen de 5 pertenecientes al estado de Jalisco que integran un núcleo de alta especialización en la industria del tequila (tres municipios centrados en Arandas y dos en Tequila). Los 10 restantes cuentan con cierta importancia individual. No obstante, la representación de estos 15 municipios es limitada pues queda por abajo del 17\% en lo económico y aloja sólo al $12 \%$ de la población.

Una vez que se integró el Bajío con las $10 \mathrm{ZM}$, la región tequilera y los 10 municipios individuales se da cuenta del 94.2\% del VA y $86.7 \%$ del empleo en la región (cuadro 1). En el mismo cuadro, también puede notarse que la participación de estas ZM y municipios importantes en la población es más moderada, sólo alcanza $71.9 \%$ del Bajío. Esto indica que la población de otros municipios del Bajío amplio está más dispersa: casi $30 \%$ se localiza en poblados de poca importancia económica que generan un reducido $17.3 \%$ del empleo formal.

Las seis ZM principales representan sin duda una gran importancia al total del Bajío: $74.7 \%$ del VA y 71.0\% del empleo. Estas incluyen a Guadalajara, Querétaro, León, San Luis Potosí, Aguascalientes y Celaya, en orden de importancia económica. Destaca la ZM Guadalajara (8 municipios) que excede de un tercio su participación en el Bajío, tanto en VA como en empleo (32.9\% y $32.7 \%$ del Bajío respectivamente). Las otras cinco importantes aportan en conjunto $41.8 \% \mathrm{y}$ $38.3 \%$ respectivamente. Otras localidades de relativa importancia son los municipios de Irapuato, San Juan del Río, Salamanca y Zacatecas (ZM), todos aportan por encima del 1\% del VA y del empleo del Bajío, así como Guanajuato, Fresnillo y Calera por su participación en el VA como se muestra en el cuadro 1. San Francisco del Rincón es un caso particular por su participación en el empleo, siendo una extensión natural de la intensidad de uso de mano de obra de la industria del calzado y curtiduría, situación que comparte también con León.

6 Dos casos quedan en el límite inferior. La ZM La Piedad-Pénjamo cuya participación se limita al $0.46 \%$ del VA de la región, aunque la ZM es conocida por su importancia agropecuaria. El otro caso es Ciudad Valles que participa del $0.48 \%$ del VA regional y del doble $(0.94 \%)$ en población. 


\section{Cuadro 1}

ZONAS METROPOLITANAS Y MUNICIPIOS IMPORTANTES DEL BAJÍO: VA, POBLACIÓN Y EMPLEO 2008 PARTICIPACIÓN PORCENTUAL

\begin{tabular}{|c|c|c|c|c|c|}
\hline ZM Y MUNICIPIOS IMPORTANTES & $\mathrm{VA}^{\mathrm{a}}$ & $L^{b}$ & $\mathrm{POB}^{\mathrm{c}}$ & POB & VA MANUF $^{d}$ \\
\hline Bajío Amplio: 210 municipios & 100.00 & 100.00 & 100.00 & 18.71 & \\
\hline ZM e importantes: 46 municipios & 94.18 & 86.73 & 71.95 & 22.55 & 48.89 \\
\hline Zonas Metropolitanas: & 77.59 & 75.51 & 59.31 & 23.82 & 47.30 \\
\hline Guadalajara (8) & 32.91 & 32.75 & 24.82 & 24.69 & 41.30 \\
\hline Querétaro (4) & 11.06 & 8.67 & 6.14 & 26.41 & 53.84 \\
\hline León (2) & 9.86 & 11.18 & 9.01 & 23.21 & 43.92 \\
\hline San Luis Potosí (2) & 9.46 & 7.96 & 5.82 & 25.59 & 53.75 \\
\hline Aguascalientes (3) & 7.41 & 6.63 & 5.22 & 23.76 & 56.61 \\
\hline Celaya (3) & 4.03 & 3.82 & 3.37 & 21.22 & 56.67 \\
\hline Zacatecas (3) & 1.01 & 1.78 & 1.73 & 19.17 & 11.35 \\
\hline Ocotlán (2) & 0.84 & 0.72 & 0.79 & 16.99 & 76.10 \\
\hline San Francisco del Rincón & 0.55 & 1.12 & 1.02 & 20.49 & 65.14 \\
\hline La Piedad-Pénjamo (2) & 0.46 & 0.89 & 1.40 & 11.94 & 31.79 \\
\hline Municipios importantes: & 15.30 & 9.71 & 10.83 & 16.77 & 55.34 \\
\hline Irapuato & 2.94 & 2.77 & 2.96 & 17.50 & 59.44 \\
\hline San Juan del Río & 2.86 & 1.62 & 1.35 & 22.46 & 61.92 \\
\hline Salamanca & 2.38 & 1.08 & 1.46 & 13.88 & 76.63 \\
\hline Guanajuato & 1.80 & 0.91 & 0.96 & 17.62 & 5.33 \\
\hline Fresnillo & 1.52 & 0.91 & 1.19 & 14.21 & 5.77 \\
\hline Calera & 1.16 & 0.31 & 0.22 & 25.59 & 82.22 \\
\hline Apaseo el Grande & 0.88 & 0.29 & 0.48 & 11.42 & 85.73 \\
\hline Lagos De Moreno & 0.65 & 0.64 & 0.86 & 13.96 & 80.07 \\
\hline San José Iturbide & 0.62 & 0.38 & 0.41 & 17.72 & 88.96 \\
\hline Ciudad Valles & 0.48 & 0.80 & 0.94 & 15.92 & 32.82 \\
\hline Región Tequilera: & 1.29 & 1.50 & 1.80 & 15.61 & 68.07 \\
\hline Tepatitlán de Morelos & 0.41 & 0.70 & 0.76 & 17.29 & 46.72 \\
\hline Tequila & 0.35 & 0.16 & 0.23 & 13.02 & 89.68 \\
\hline Arandas & 0.21 & 0.35 & 0.41 & 15.97 & 63.12 \\
\hline Amatitán & 0.17 & 0.06 & 0.08 & 14.46 & 81.17 \\
\hline Atotonilco el alto & 0.16 & 0.23 & 0.32 & 13.30 & 68.59 \\
\hline
\end{tabular}

Notas: a) Participación en VA al Bajío Amplio. b) Participación en el empleo (L) al Bajío Amplio. c) Participación en la población al Bajío Amplio. d) Participación en VA de las manufacturas en el total de VA de la ZM o municipio.

Fuente: Elaborado con datos de los Censos Económicos y Censo de Población y Vivienda 2010 (INEGI, 2009 y 2010).

El cuadro 1 también anticipa la vocación manufacturera en general. En las ZM importantes la proporción de VA manufacturero oscila entre 41 y $57 \%$. En estas ZM la manufactura se reparte entre varias actividades de importancia, como se verá más adelante al tratar los índices 
de concentración. En cambio, los municipios individuales suelen estar económicamente dominados por una o dos industrias; es el caso muy evidente de Ocotlán (y Poncitlán), San Francisco del Rincón (y Purísima), Irapuato, Salamanca, Calera, Apaseo el Grande, San José Iturbide, Lagos de Moreno, y la región Tequilera, todos con participación de manufacturas por encima de 59\% del VA.

En la siguiente sección se caracterizan las ZM y los municipios por actividades económicas destacadas que determinarán las condiciones competitivas del Bajío.

\section{ACTIVIDADES IMPORTANTES DEL BAJío}

La actividad económica del Bajío se compone de 82 actividades que incluyen subsectores, ramas y clases censales (3, 4 y 6 dígitos) según lo justifique su importancia y sus características individuales 7 . En total, esas 82 actividades representan $95.2 \%$ de la actividad productiva del Bajío, la mayoría de ellas (74 actividades) alcanzando más del $0.5 \%$ del valor agregado de la región. Como se observa en el cuadro 2, son pocas las actividades importantes nacionalmente que no tienen presencia en el Bajío, entre ellas destacando la extracción de petróleo, la actividad

Cuadro 2

ACTIVIDADES IMPORTANTES EN EL BAJíO Y EN EL AGREGADO NACIONAL, 2008

\begin{tabular}{|c|c|c|c|}
\hline & \multicolumn{3}{|c|}{$\% \mathrm{VA}$} \\
\hline ACTIVIDADES POR IMPORTANCIA & \# & BAJÍO & NACIONAL \\
\hline \multicolumn{4}{|l|}{ A. Comparación entre Bajío y el país } \\
\hline Importantes en Bajío y Nacional & (79) & 92.72 & $70.55^{\mathrm{a}}$ \\
\hline De cierta importancia en Bajío & (3) & 2.49 & 1.52 \\
\hline Suma Total & $(82)$ & 95.21 & 72.07 \\
\hline \multicolumn{4}{|l|}{ B. Actividades importantes en Bajío } \\
\hline Muy importantes & (6) & 17.34 & \\
\hline Importantes & (14) & 24.16 & \\
\hline Medianamente importantes & (8) & 10.86 & \\
\hline Poco importantes & (54) & 42.85 & \\
\hline Suma Total & $(82)$ & 95.21 & \\
\hline
\end{tabular}

Notas: a) Las tres actividades de mayor importancia en el agregado nacional son la extracción de petróleo (19.9\%), los Corporativos (1.8\%) y las Casas de Bolsa (1.3\%), sumando $23.0 \%$. Estas actividades no se encuentran en el Bajío. Ver Unger, 2014b.

Fuente: Elaborado con datos de los Censos Económicos 2009 (INEGI, 2009).

Esta sección sigue de cerca a la descripción de importancia y vocaciones productivas del Bajío en la sección correspondiente de Unger et al.(2014a). 
de corporativos y las casas de bolsa; estas tres actividades aportan en conjunto el $23 \%$ del VA nacional.

La importancia individual de cada actividad en el Bajío es moderada, no hay ninguna que se asemeje ni remotamente a la representación de $19.9 \%$ que alcanza el petróleo en la cuenta nacional. Las seis actividades más importantes en el Bajío suman 17.3\% del total, proporción semejante al comparativo nacional sin incluir al petróleo. Estas actividades principales en el Bajío son electricidad, autos y camiones (ambas por encima de 3\%), seguidas por telecomunicaciones alámbricas, comercio de materias primas- mayoreo, abarrotes- mayoreo y productos metálicos (todas por arriba de $2 \%$, ver gráfica 1). Algunas de estas actividades alcanzan presencia muy notable en ciertas localidades, como es el caso de la energía y las telecomunicaciones para Guanajuato, así como los autos para Aguascalientes y León.

\section{Gráfica 1}

ACTIVIDADES IMPORTANTES (VA $>1.2 \%{ }^{\mathrm{a}}$ ) EN EL BAJÍO Y LA IMPORTANCIA QUE ALCANZAN EN ALGUNA ZM O MUNICIPIO (VA > 13\% EN ZM), 2008

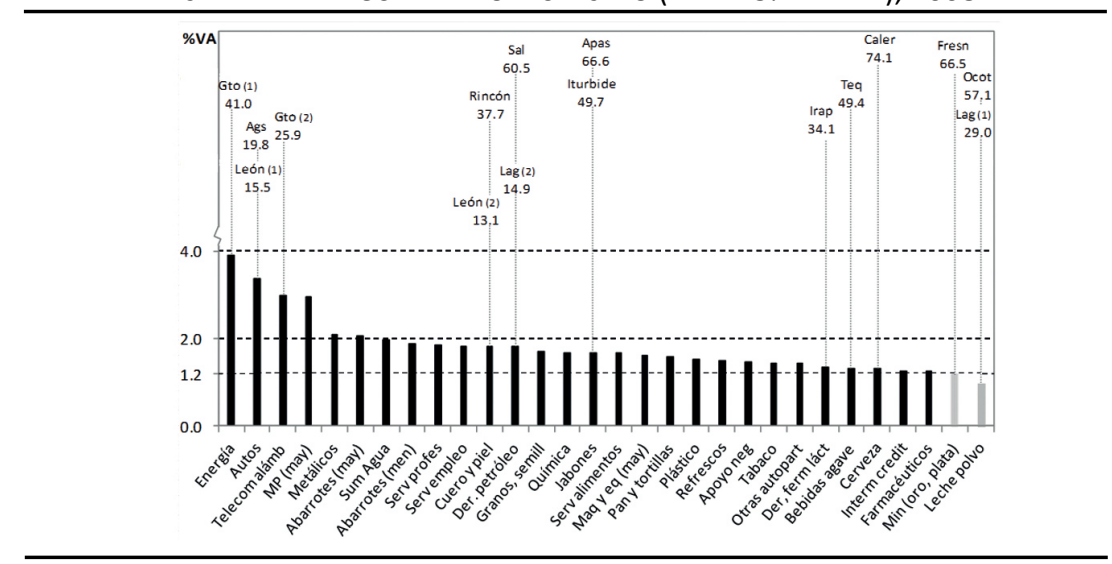

Nota: a) Minería (oro y plata) y Leche en polvo no cumplen el criterio de importancia en Bajío (\%VA>1.2), pero se agregan por ser actividades de mucha importancia en algunos municipios.

Fuente: Elaborado con datos de los Censos Económicos 2009 (INEGI, 2009).

A partir de ahí, las actividades en esa gráfica se mantienen en rangos de importancia moderada entre 1.2 y $2 \%$, por lo que la suma de las 28 mayores representa el 52.4\% del total de la Región (ver Anexo II en Unger et al, 2014a). En ellas se incluyen otras catorce actividades mayores a $1.5 \%$ de muy diversa naturaleza, tales como suministro de agua, abarrotes y bebidas-menudeo, servicios profesionales, servicios 
de empleo, curtido y productos de cuero y piel, derivados del petróleo, aceites y grasas, servicios de alimentos y bebidas, jabones y limpiadores, comercio de maquinaria y equipo-mayoreo, pan y tortillas, química, plástico y producción de refrescos. Tres de estas actividades representan una gran importancia a la producción de ciertas localidades, es el caso de cuero y piel para las ZM de León y Rincón, derivados de petróleo para Salamanca y Lagos y jabones y limpiadores de notable presencia en Apaseo el Grande y San José Iturbide. En estos últimos la dominancia de una sola empresa es evidente.

Otras ocho actividades por encima del $1.2 \%$ son bebidas destiladas de agave, derivados y fermentos lácteos, tabaco, autopartes (otras), farmacéuticos, cerveza, intermediación crediticia y servicios de apoyo a negocios. También destacan tres de estas actividades por su importancia a la producción de un municipio en cada caso, a saber, derivados lácteos en Irapuato, agave en la región Tequilera y cerveza en Calera, Zacatecas.

Estos dos grupos de 22 actividades con representación entre 1.2 y $2 \%$ son más interesantes por tratarse en su mayoría de rubros estrictamente comerciables y competidos que deben sostenerse por competitividad propia. El contraste es notable con el primer grupo, como pudimos observar, en el que predominan actividades de servicios (tanto públicos como privados) y comercios ${ }^{8}$. Por último, en la gráfica 1 se agregan otras dos actividades que alcanzan mucha importancia en municipios particulares: minería de oro y plata (Fresnillo) y leche en polvo (Ocotlán y Lagos de Moreno).

Como se anticipó en la sección 1, la caracterización agregada del Bajío a nivel sectorial destaca la vocación manufacturera por sobre las demás (49.6\% del VA), aunque secundariamente el comercio también sobresale en ciertas localizaciones (cuadro 3). Las ZM importantes con mayor propensión a la manufactura son Querétaro, San Luis Potosí, Aguascalientes y Celaya, las cuatro con porcentajes superiores a 54\% del VA. En cambio la ZM Guadalajara destaca por el comercio, en que alcanza $22.5 \%$, superando la media del Bajío de $17.7 \%$ y la media

8 La distinción entre comerciables y no comerciables (tradeables / non tradeables, que pueden traducirse también como transables / no transables si se prefiere) es crucial para nuestros fines de asociar importancia con especialización y condiciones de competitividad. La clasificación de actividades por esa dicotomía la hicimos en ocasión de otro trabajo (ver Unger, 2009). 


\section{Cuadro 3}

PARTICIPACIÓN EN VALOR AGREGADO DE LAS 82 ACTIVIDADES POR SECTORES, COMERCIABILIDAD Y ZM O MUNICIPIOS, 2008

\begin{tabular}{|c|c|c|c|c|c|c|c|c|}
\hline & \multirow[b]{2}{*}{ MANUF } & \multicolumn{3}{|c|}{ SECTORES } & \multicolumn{3}{|c|}{ COMERCIABILIDAD } & \multirow[b]{2}{*}{$\begin{array}{c}\% \mathrm{VA} \\
\text { 82ACT/TOT }\end{array}$} \\
\hline & & COM & SERV & INF & MIN & $\mathrm{C}$ & NC & \\
\hline Nacional $^{a}$ & 38.2 & 16.8 & 34.3 & 10.3 & 0.4 & 34.7 & 65.3 & \\
\hline Bajío & 49.6 & 17.7 & 22.1 & 9.3 & 1.2 & 46.8 & 53.2 & 95.2 \\
\hline \multicolumn{9}{|l|}{ Zonas Metropolitanas } \\
\hline Guadalajara (8) & 41.9 & 22.5 & 24.6 & 11.0 & & 39.7 & 60.3 & 93.9 \\
\hline Querétaro (4) & 54.4 & 16.8 & 24.4 & 4.4 & & 46.3 & 53.7 & 97.0 \\
\hline León (2) & 44.9 & 17.5 & 32.4 & 5.3 & & 42.8 & 57.2 & 95.8 \\
\hline San Luis Potosí (2) & 54.3 & 13.3 & 20.8 & 11.6 & 0.0 & 47.1 & 52.9 & 95.1 \\
\hline Aguascalientes (3) & 57.1 & 15.0 & 16.5 & 11.4 & & 50.1 & 49.9 & 94.7 \\
\hline Celaya (3) & 59.6 & 16.1 & 19.7 & 4.6 & & 55.3 & 44.7 & 94.4 \\
\hline Zacatecas (3) & 12.5 & 38.6 & 31.3 & 17.6 & 0.0 & 14.6 & 85.4 & 87.3 \\
\hline Ocotlán (2) & 76.3 & 13.2 & 8.6 & 1.8 & & 73.6 & 26.4 & 92.9 \\
\hline Sn Fco del Rincón (2) & 63.8 & 21.9 & 11.8 & 2.4 & & 57.1 & 42.9 & 93.4 \\
\hline Piedad-Pénjamo (2) & 31.9 & 35.4 & 29.5 & 3.1 & & 29.0 & 71.0 & 96.5 \\
\hline \multicolumn{9}{|l|}{ Municipios Importantes } \\
\hline Irapuato & 60.6 & 22.2 & 12.0 & 5.2 & & 51.5 & 48.5 & 97.3 \\
\hline San Juan del Río & 60.4 & 9.0 & 10.5 & 20.2 & & 58.9 & 41.1 & 94.4 \\
\hline Salamanca & 77.5 & 7.7 & 11.4 & 3.3 & & 80.9 & 19.1 & 98.7 \\
\hline Guanajuato & 5.4 & 5.8 & 35.9 & 45.8 & 7.1 & 12.2 & 87.8 & 98.7 \\
\hline Fresnillo & 5.8 & 12.8 & 6.8 & 8.1 & 66.5 & 71.8 & 28.2 & 97.0 \\
\hline Calera & 82.4 & 1.8 & 15.5 & 0.4 & & 79.2 & 20.8 & 99.8 \\
\hline Apaseo el Grande & 86.0 & 1.3 & 12.4 & 0.3 & & 83.8 & 16.2 & 99.4 \\
\hline Lagos De Moreno & 80.8 & 13.0 & 5.5 & 0.7 & & 74.3 & 25.7 & 97.7 \\
\hline San José Iturbide & 90.5 & 5.6 & 3.5 & 0.4 & & 58.7 & 41.3 & 97.4 \\
\hline Ciudad Valles & 34.4 & 41.8 & 18.2 & 5.5 & & 19.4 & 80.6 & 94.6 \\
\hline Región Tequilera & 68.5 & 18.5 & 12.0 & 1.0 & & 68.3 & 31.7 & 97.9 \\
\hline
\end{tabular}

Notas: a) No se incluyen otras actividades no presentes en Bajío. Destacarán en esas, la extracción de petróleo y muchos de los servicios de mayor importancia nacional.

Fuente: Elaborado con datos de los Censos Económicos 2009 (INEGI, 2009).

nacional de $16.8 \%$ (ver cuadro 3). También es diferente la ZM León que presenta la mayor inclinación por el sector servicios (32.4\% comparado al $22.1 \%$ del Bajío conjunto), aunque todavía a distancia de otras entidades como el DF (actualmente Ciudad de México), cuya tendencia a la economía de servicios ha empujado la participación de éstos a $34.3 \%$ en el agregado nacional. En el mismo sentido, dada la predominancia de actividades manufactureras entre las comerciables, encontramos que los sectores de comerciables tienden a ser menores al promedio 
del Bajío en las localidades más orientadas al comercio, tales como la ZM Guadalajara y ZM León, así como Zacatecas, La Piedad-Pénjamo, Guanajuato y Ciudad Valles. Estas últimas quedan muy rezagadas aún respecto de la media nacional de comerciables

\section{Gráfica 2}

ÍNDICES DE CONCENTRACIÓN DE \%VA EN LAS 21 ZONAS METROPOLITANAS Y MUNICIPIOS IMPORTANTES, 2008

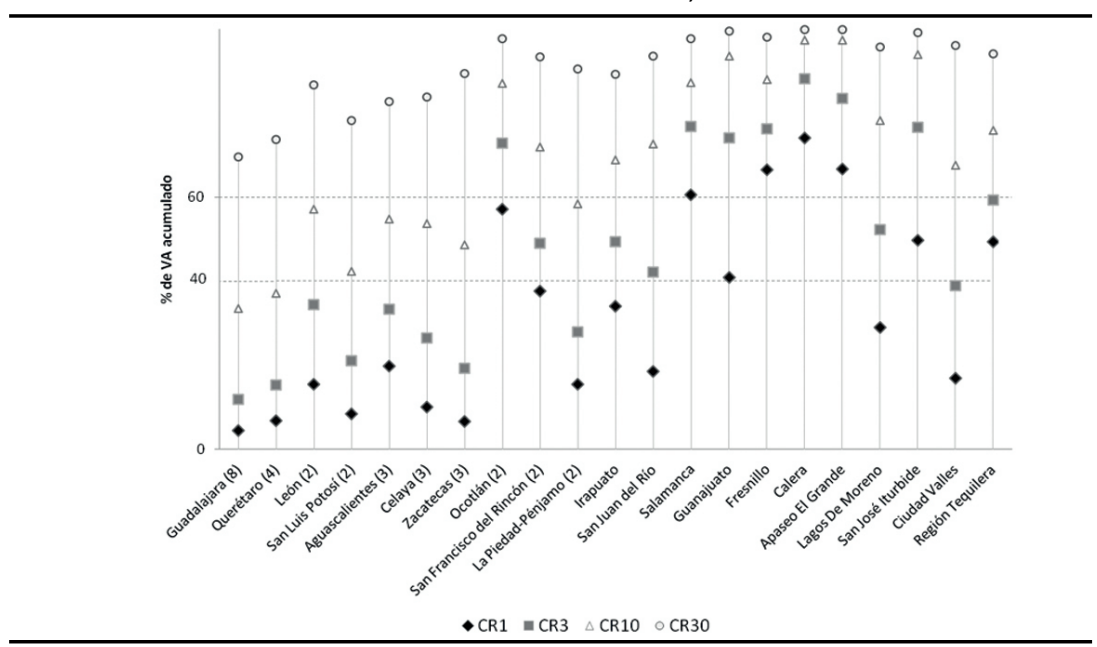

Fuente: Elaborado con datos de los Censos Económicos 2009 (INEGI, 2009).

Otras vocaciones más singulares alcanzan a la ZM Zacatecas, de marcada orientación a comercio y servicios, y como ya se anticipó, las manufacturas alcanzan muy alta participación en localidades más pequeñas como las ZM Ocotlán y San Francisco del Rincón, además de los municipios de Irapuato, San Juan del Río, Salamanca, Calera, Apaseo el Grande, Lagos de Moreno, San José Iturbide y la Región Tequilera. Como también se dijo antes, y veremos con más detalle al analizar la competitividad, estas localidades suelen estar muy dominadas por la importancia de una o dos actividades particulares. Es el mismo caso a destacar en la influencia de la minería en Fresnillo, impuesta por la empresa Peñoles. Una clara indicación de esto se obtiene de los índices de concentración que analizaremos enseguida.

El resultado más general hasta ahora, que va de la mano con lo sectorial, es que las actividades comerciables destacan en la mayoría de las ZM y municipios de importancia. En casi todos los casos en que las 
comerciables superan a las no comerciables se aprecia el impacto de la actividad más importante de la localidad, como podemos observar en la estadística del CR1 en el cuadro 4. La excepción a destacar es San Juan del Río cuya actividad principal es no comerciable (energía eléctrica), aunque las dos siguientes en importancia se le semejan y son comerciables, lo que provoca un CR3 que se alinea a la tendencia general (ver gráfica 2).

Las estimaciones de concentración del cuadro 4 y la gráfica 2 se complementan para ilustrar estas tendencias y servirán también al análisis siguiente en que se busca identificar los casos de mejor situación de competitividad compartida.

\section{LA COMPETITIVIDAD DE LAS ZM Y MUNICIPIOS DEL BAJÍO: UNA DEFINICIÓN POR VENTAJAS REVELADAS DE ACTIVIDADES IMPORTANTES ${ }^{9}$}

En esta sección se estima primero la competitividad asociada a las actividades importantes de cada ZM y municipio. En una segunda instancia se busca comprobar la relación entre dicha competitividad agregada y las medidas de concentración/dispersión de las actividades. El propósito es poner a prueba la idea de que hay mejores ventajas competitivas en una zona cuando las competitividades son compartidas entre más actividades de los espacios cercanos de las ZM y los municipios.

La noción de competitividad que se aplica distingue a las buenas prácticas de aumento de la productividad laboral acompañadas por mayores salarios y acopio de capital humano. La definición de competitividad toma diferentes significados según el ámbito geográfico o de política económica al que se quiere referir $^{10}$. La interpretación que en este trabajo se presenta asume la competitividad como la capacidad de una economía para atraer y desarrollar empresas de alta producti-

9 La introducción conceptual a la competitividad de acuerdo con el principio de ventajas reveladas puede consultarse en Unger (2012).

10 Otras acepciones refieren a participación en el mercado y a diversas medidas de territorio. Con relación a la competitividad territorial, la idea de externalidades benéficas al conjunto ha venido imponiendo el término de ventajas competitivas de una región, como lo definen Schwab and Porter (2007): "Regional competitiveness is the set of institutions, policies and factors that determine the level of productivity of a region. The level of productivity, in turn, sets the sustainable level of prosperity that can be earned by an economy". 
vidad, a la vez que asegura de que los niveles de vida de sus pobladores mejoran en el tiempo (Huggins y Izushi, 2008).

El marco de referencia más amplio al tema de la competitividad puede tomar elementos adicionales de las teorías de la organización industrial contemporánea, la nueva geografía económica, la nueva teoría de comercio y las vertientes de desarrollo más de tipo institucional. De ellas pueden provenir aspectos de relevancia que le asocian a la competitividad no sólo los atributos de productividad y capital humano a los que nosotros damos prioridad, sino también el análisis de estructuras de mercado, patrones de especialización, dominio de espacios territoriales y grados de comerciabilidad aplicables a distintas actividades, aspectos todos que pueden o no contribuir al bienestar de la población. Esta literatura que integra competitividad con estructuras de competencia en entornos microespecíficos de geografía, puede incluir una variedad de autores con matices teóricos diferentes, tales como Krugman (1996), Porter (1990) y Rodrik (2007).

Con estos antecedentes, nos proponemos cuantificar la competitividad de las ZM y los municipios del Bajío a través de un índice relativamente simple que recoge tanto la productividad laboral, como la derrama de las ganancias por productividad reflejada en mayores salarios. En otras palabras, se toma como prioridad la versión de competitividad de tipo económico y no aquéllas de características organizacionales o institucionales, que también pueden ser útiles según muestran otros ejercicios ${ }^{11}$.

La siguiente es una medida de competitividad ${ }^{12}$ de actividades líderes en cada ZM o municipio recuperada de Unger (2014c):

$$
\mathrm{C}_{\mathrm{act}}^{* *}=\left(\frac{\mathrm{VA}}{\mathrm{L}}\right)^{*}+\left[\left(\frac{\mathrm{W}}{\mathrm{L}}\right)^{*}-1\right]=\left[\frac{\left(\frac{\mathrm{VA}}{\mathrm{L}}\right)_{\mathrm{act}, \mathrm{zM}}}{\left(\frac{\mathrm{VA}}{\mathrm{L}}\right)_{\text {act, Pais }}}\right]+\left[\left(\frac{\left(\frac{\mathrm{W}}{\mathrm{L}}\right)_{\mathrm{act}, \mathrm{zM}}}{\left(\frac{\mathrm{W}}{\mathrm{L}}\right)_{\text {act, Pais }}}\right)-1\right]
$$

11 Es por demás evidente que hay diversas maneras de acercarse al tema de la competitividad en una localidad. Algunos prefieren ocuparse de las características del entorno legal, institucional, de gobernabilidad y políticas públicas que pueden ser sin duda también relevantes, sobre todo al comparar el atractivo para los inversionistas entre diversas localidades (Cabrero et al., 2009; Imco, 2011; EGAP, 2011). Ver la revisión comparativa en curso de Garduño et al. (2013).

12 Cada uno de los componentes refleja si el valor para la actividad local es mayor (menor) que la nacional cuando el cociente es mayor (menor) que la unidad. El índice global es igual a uno cuando tanto la productividad como los salarios son iguales en esa localidad y en el país. 
$(\mathrm{VA} / \mathrm{L})^{*}=$ Ventaja por productividad

$(\mathrm{W} / \mathrm{L})^{*}=$ Ventaja por salario.

El mejor resultado anticipado puede ser la ganancia de competitividad $\left(\mathrm{C}^{* *}\right)$ por contribución positiva de ambos factores y será aún mejor si proviene de las actividades comerciables expuestas a competencia. Todos los valores de $\mathrm{C}^{* *}$ superiores a la unidad denotan competitividad. La primera observación a destacar es que los resultados del cuadro 4 confirman que el Bajío en general padece de poca competitividad, tanto por productividad como por nivel salarial ${ }^{13}$. Sólo en tres localidades la media aritmética de todas las actividades alcanza condición competitiva: Querétaro $\left(\mathrm{C}^{* *}=1.17\right)$, Guadalajara $\left(\mathrm{C}^{* *=1.01}\right)$ e Irapuato $\left(\mathrm{C}^{* *=1: 00)}\right.$. Únicamente Querétaro mantiene registros incuestionables, con ganancias de productividad lo mismo que salariales, y también al agrupar las actividades en comerciables por igual que en no comerciables. Guadalajara e Irapuato solamente alcanzan competitividad en actividades no comerciables (columnas 5 y 6 de cuadro 4). El resto de las ZM y municipios del Bajío caen muy por debajo en ambos indicadores, lo cual se refleja en una media de competitividad $\mathrm{C}^{* *}=0.46$ para las 18 localidades restantes. La tendencia se repite con muy pocas variantes y una sola excepción que es Apaseo el Grande por la influencia de una de sus actividades comerciables ${ }^{14}$ (cuadro 4). Aún así, estas son medias aritméticas que no necesariamente aluden a una competitividad sistémica o compartida, pues es frecuente que esas medias estén muy impactadas por pocas actividades como ilustraremos enseguida.

El listado de las ZM y municipios en el cuadro 4 muestra una extensa heterogeneidad de condiciones competitivas. Prevalece pues la idea de un Bajío sumamente heterogéneo en cuanto a competitividad. Aún así y a pesar de las diferencias manifiestas entre las localidades, la

13 Entre los principales resultados, debemos advertir que el Bajío en general padece de poca competitividad, tanto por productividad como por nivel salarial. Ante tal premisa será importante considerar si hay algunos indicios promisorios del desempeño en el tiempo de estos dos factores de la competitividad, pues puede haber localidades en movimiento económico y no otras que están condenadas a ser sitios de alojamiento de poblaciones crecientes sin actividad propia.

14 Se trata de 13 actividades comerciables en Apaseo cuya $C^{* *}$ media se impacta desmedida-

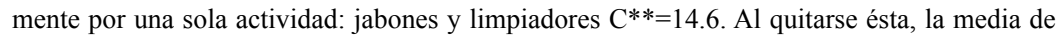
competitividad quedaría en un pobre 0.13 . 
correlación de rango entre la importancia económica (\%VA) de cada localidad y las medidas de competitividad y productividad prueba ser robusta y significativa, lo que aporta un cierto grado de congruencia en medio del clima desfavorable en general. La significancia se respeta también para los grupos de comerciables y no comerciables separadamente. Sin embargo, las generalizaciones no valen, por lo que intentamos precisar el papel de las actividades individualmente en cada localidad y su potencial de mejorar en el futuro.

La media aritmética de $\mathrm{C}^{* *}$ de todas las actividades de cada localidad puede esconder algunas situaciones extrañas o engañosas que le restan representatividad a esa medida como expresión de competitividad sistémica o de conjunto. En ocasiones, el valor de una sola actividad puede distorsionar la interpretación de la media como representante de todas. Por tal motivo, la estimación más precisa de condiciones competitivas sistémicas se hace deduciendo la actividad principal (importancia neta: \%VAcomp - CR1 comp) e incorporando otros criterios acerca de la diversificación o alcance de las condiciones competitivas, los cuales se definen en el siguiente apartado.

\section{Cuadro 4}

COMPETITIVIDAD, PRODUCTIVIDAD Y SALARIOS POR ZM Y MUNICIPIOS IMPORTANTES DEL BAJIOO: ORDENAMIENTO POR IMPORTANCIA Y CORRELACIONES, 2008

\begin{tabular}{|c|c|c|c|c|c|c|}
\hline \multirow{2}{*}{$\begin{array}{c}\text { ZONAS METROPOLITANAS Y } \\
\text { MUNICIPIOS }\end{array}$} & \multirow{2}{*}{$\% \mathrm{VA}^{\mathrm{a}}$} & \multicolumn{3}{|c|}{82 ACTS } & \multirow{2}{*}{$\begin{array}{c}\mathrm{C} \\
\mathrm{C}^{* *}\end{array}$} & \multirow{2}{*}{$\begin{array}{l}\mathrm{NC} \\
\mathrm{C}^{* *}\end{array}$} \\
\hline & & $(\mathrm{VA} / \mathrm{L})^{*}$ & $(W / L)^{*}$ & $\mathrm{C}^{* *}$ & & \\
\hline \multicolumn{7}{|l|}{ Zonas Metropolitanas } \\
\hline Guadalajara (8) & 34.46 & 0.98 & 1.03 & 1.01 & 0.85 & 1.16 \\
\hline Querétaro (4) & 11.97 & 1.08 & 1.07 & 1.17 & 1.10 & 1.23 \\
\hline León (2) & 10.53 & 0.76 & 0.91 & 0.70 & 0.39 & 0.93 \\
\hline San Luis Potosí (2) & 10.03 & 0.87 & 0.88 & 0.75 & 0.45 & 0.98 \\
\hline Aguascalientes (3) & 7.83 & 0.86 & 0.93 & 0.81 & 0.74 & 0.87 \\
\hline Celaya (3) & 4.25 & 0.77 & 0.84 & 0.65 & 0.56 & 0.70 \\
\hline Zacatecas (3) & 0.99 & 0.48 & 0.65 & 0.16 & -0.22 & 0.37 \\
\hline Ocotlán (2) & 0.87 & 0.64 & 0.68 & 0.34 & 0.19 & 0.43 \\
\hline Sn Fco del Rincón (2) & 0.57 & 0.65 & 0.66 & 0.27 & -0.07 & 0.41 \\
\hline Piedad-Pénjamo (2) & 0.49 & 0.54 & 0.62 & 0.19 & -0.10 & 0.32 \\
\hline \multicolumn{7}{|l|}{ Municipios Importantes } \\
\hline Irapuato & 3.19 & 1.08 & 0.90 & 1.00 & 0.92 & 1.05 \\
\hline San Juan Del Río & 3.02 & 0.89 & 0.93 & 0.84 & 0.88 & 0.81 \\
\hline Salamanca & 2.62 & 0.79 & 0.70 & 0.53 & 0.47 & 0.55 \\
\hline
\end{tabular}


Cuadro 4 (CONTINUACIÓN)

\begin{tabular}{|c|c|c|c|c|c|c|}
\hline $\begin{array}{l}\text { ZONAS METROPOLITANAS Y } \\
\text { MUNICIPIOS }\end{array}$ & $\% \mathrm{VAa}$ & $(\mathrm{VA} / \mathrm{L})^{*}$ & $\begin{array}{l}82 \text { ACTS } \\
(W / L)^{*}\end{array}$ & $\mathrm{C}^{* *}$ & $\begin{array}{c}\mathrm{C} \\
\mathrm{C}^{* *}\end{array}$ & $\begin{array}{l}\mathrm{NC} \\
\mathrm{C}^{* *}\end{array}$ \\
\hline Guanajuato & 1.98 & 0.70 & 0.85 & 0.59 & 0.22 & 0.68 \\
\hline Fresnillo & 1.65 & 0.69 & 0.73 & 0.50 & 0.32 & 0.56 \\
\hline Calera & 1.29 & 0.61 & 0.61 & 0.24 & 0.11 & 0.33 \\
\hline Apaseo el Grande & 0.97 & 0.73 & 0.65 & 0.41 & 1.25 & 0.04 \\
\hline Lagos De Moreno & 0.71 & 0.61 & 0.81 & 0.44 & 0.41 & 0.45 \\
\hline San José Iturbide & 0.67 & 0.60 & 0.57 & 0.20 & -0.08 & 0.33 \\
\hline Ciudad Valles & 0.51 & 0.59 & 0.74 & 0.36 & -0.30 & 0.62 \\
\hline Región Tequilera (5) & 1.41 & 0.52 & 0.69 & 0.22 & 0.24 & 0.28 \\
\hline Media aritmética & & 0.74 & 0.78 & 0.54 & 0.40 & 0.62 \\
\hline$(\mathrm{VA} / \mathrm{L}) *$ y $(\mathrm{W} / \mathrm{L}) *$ & & & $.7893 * * *$ & & $0.8157^{* * *}$ & $0.7955^{* * *}$ \\
\hline \%VA y $(\mathrm{VA} / \mathrm{L}) *$ & Corr Rango ${ }^{\mathrm{b}}$ & & $.8090 * * *$ & & $0.6234 * * *$ & $0.7796 * * *$ \\
\hline$\% \mathrm{VA}$ y $\mathrm{C}^{* *}$ & & & $8455^{* * *}$ & & $0.7026^{* * *}$ & $0.8035^{* * *}$ \\
\hline
\end{tabular}

Notas: a) Participación en VA que aporta cada ZM o municipio importante a la Región Bajío (46 municipios). b) Correlación de rango significativa ( $* * *: 1 \%$ de significancia).

Fuente: Elaborado con datos de los Censos Económicos 2009 (INEGI, 2009).

\subsection{Competitividad sistémica: grado de diversificación}

En esta sección se profundiza en el análisis de la competitividad sistémica de la región. El propósito es comprobar que las mejores ventajas competitivas en una localidad se obtienen cuando existe una proporción alta de actividades con $\mathrm{C}^{* *}$ mayor que la unidad. En otras palabras, la competitividad sistémica real se da cuando las condiciones competitivas se comparten entre muchas de las actividades de una localidad. El análisis de la competitividad sistémica se compone de dos aspectos: la importancia y el grado de diversificación de las actividades competitivas.

La importancia para cada localidad se ha definido por las medidas del número de actividades competitivas, la importancia neta tal como fue definida antes, y la proporción del empleo (\%Lcomp) que representan esas actividades ${ }^{15}$.

Para evaluar la competitividad sistémica del conjunto de localidades se implementa un cluster analysis con las variables \%VAcomp - CR1 comp

15 A la proporción de empleo (L) no se le resta la actividad principal, dado que su impacto en el empleo suele ser relativamente menor. Las distorsiones mayores sin duda provienen de la medida desproporcionada de VA. 
y \%Lcomp con el fin de identificar subgrupos que compartan condiciones competitivas similares. En el cuadro 5 columna 6, se plasman los resultados más significativos del cluster analysis. Hay tres grupos o clusters de localidades en cuanto a la importancia económica y la importancia del empleo en las actividades competitivas. El grupo de pertenencia al cluster 1 es significativamente más competitivo que los otros dos. Es indicativo de ciudades o ZM que han llegado a reunir

\section{Cuadro 5}

CLUSTER ANALYSIS DE ZM Y MUNCIPIOS IMPORTANTES POR PARTICIPACIÓN DE LAS ACTIVIDADES COMPETITIVAS EN VA Y EMPLEO, 2008

\begin{tabular}{|c|c|c|c|c|c|c|}
\hline ZM O MUNICIPIO & \#ACTS COMP & $\%$ VA COMP & CR1 COMP & $\begin{array}{l}\text { \%VA COMP- } \\
\text { CR1 COMP }\end{array}$ & $\%$ L COMP & $\begin{array}{c}\text { CLUSTER \%VA-CR1 } \\
\text { COMP Y \% } \text { L }^{\mathrm{a}-}\end{array}$ \\
\hline \multicolumn{7}{|l|}{ GRUPO 1} \\
\hline Querétaro (4) & 51 & 81.7 & 6.8 & 74.9 & 79.4 & 1 \\
\hline Aguascalientes (3) & 30 & 74.9 & 19.8 & 55.1 & 49.4 & 1 \\
\hline León (2) & 27 & 70.8 & 15.5 & 55.3 & 62.6 & 1 \\
\hline Celaya (3) & 20 & 61.3 & 10.0 & 51.3 & 41.5 & 1 \\
\hline Guadalajara (8) & 38 & 58.9 & 4.4 & 54.5 & 57.8 & 1 \\
\hline San Luis Potosí (2) & 29 & 48.4 & 3.8 & 44.6 & 51.5 & 1 \\
\hline \multicolumn{7}{|l|}{ GRUPO 2} \\
\hline Salamanca & 17 & 88.9 & 60.5 & 28.4 & 32.2 & 2 \\
\hline San José Iturbide & 9 & 79.2 & 49.7 & 29.5 & 34.7 & 2 \\
\hline Irapuato & 21 & 68.0 & 34.1 & 33.9 & 43.3 & 2 \\
\hline Ciudad Valles & 12 & 64.2 & 17.0 & 47.2 & 27.8 & 2 \\
\hline Lagos De Moreno & 13 & 58.0 & 28.9 & 29.1 & 28.7 & 2 \\
\hline San Juan del Río & 20 & 56.6 & 13.1 & 43.5 & 29.6 & 2 \\
\hline Zacatecas (3) & 10 & 34.7 & 6.6 & 28.1 & 26.5 & 2 \\
\hline \multicolumn{7}{|l|}{ GRUPO 3} \\
\hline Apaseo el Grande & 5 & 84.1 & 66.7 & 17.4 & 9.6 & 3 \\
\hline Fresnillo & 12 & 78.8 & 66.5 & 12.3 & 33.5 & 3 \\
\hline Ocotlán (2) & 9 & 69.3 & 57.1 & 12.2 & 15.5 & 3 \\
\hline Guanajuato & 13 & 41.3 & 25.9 & 15.4 & 37.4 & 3 \\
\hline $\begin{array}{l}\text { La Piedad-Pénjamo } \\
\text { (2) }\end{array}$ & 8 & 22.4 & 5.1 & 17.3 & 27.9 & 3 \\
\hline Calera & 7 & 22.1 & 10.8 & 11.3 & 25.9 & 3 \\
\hline Región Tequilera (5) & 6 & 12.4 & 3.7 & 8.7 & 18.6 & 3 \\
\hline Sn Fo del Rincón (2) & 4 & 9.6 & 4.7 & 4.9 & 9.1 & 3 \\
\hline
\end{tabular}

Fuente: Elaboración propia con base en datos de Censos Económicos 2009 (INEGI).

Nota: a) El cluster analysis se implementa con las columnas 5 y 6 conjuntamente 
condiciones competitivas favorables y que pueden compartirse o transmitirse entre un mayor número de sus actividades. La gráfica 3 expresa a ambos indicadores señalando de nueva cuenta la competitividad más ampliamente compartida de Querétaro, León, Guadalajara, Aguascalientes, Celaya y San Luis Potosí, en ese orden y por mucho, con Querétaro a la cabeza. Estas localidades integran un grupo muy reducido y disperso con condiciones atractivas para ciertas inversiones y con ciertas restricciones o inclinaciones heredadas que trataremos de evidenciar.

Un caso muy diferente, por ser mucho menos promisorias, lo componen la mayoría de las otras localidades. La idea ingenua de extrapolación del desarrollo de la región Bajío por contagio entre las actividades y las localidades es falaz. En particular, para localidades pequeñas es común encontrar pocas actividades competitivas, aunque una de ellas muy sobresaliente puede disparar la estimación media de la competitividad económica como ya anticipamos. En la mayoría de esos casos no se puede confiar en que se darán automáticamente amplias derramas. Más bien, la estimación de la importancia neta sin incluir el impacto de la actividad principal (columna 4 del cuadro 5) da una idea más real del alcance modesto que ha logrado la competitividad sistémica. Esto aplica singularmente para Salamanca, San José Iturbide,

\section{Gráfica 3}

CLUSTER DE LOCALIDADES POR PARTICIPACIÓN EN VA Y EMPLEO DE ACTIVIDADES COMPETITIVAS, 2008

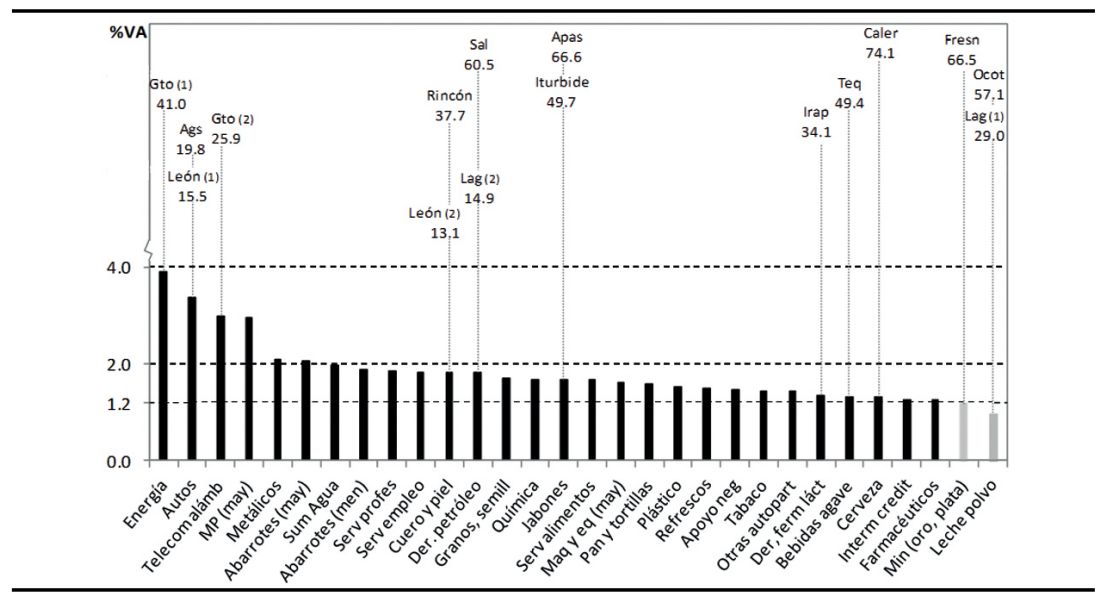

Fuente: Elaboración propia con base en datos de Censos Económicos 2009 (INEGI).

Notas: a) Se excluye la actividad principal. 
Irapuato, Apaseo el Grande, Ocotlán y Fresnillo como ya anticipamos. Ocurre también cuando consideramos el empleo, y aún más si consideráramos su calidad. Al sumar a la medición de producción también la medida de empleo competitivo, se ajusta la condición de poco peso sistémico de las actividades competitivas de Ciudad Valles y San Juan del Río por su limitado impacto en el empleo.

El otro criterio que complementa el análisis de competitividad sistémica es el grado de diversificación de las actividades competitivas. Esta es una estimación más explícita de la diversificación y se estima a partir de una modificación del índice Shannon de entropía, al cual nos referiremos como índice de entropía ajustado.

El índice de entropía ajustado (SEI*) de cada localidad se expresa como:

$$
S E I^{*}{ }_{i}=\frac{S E I_{i}}{\ln \left(n_{i}\right)} \cdot \frac{n_{i}}{N_{i}} \cdot 100
$$

donde $\quad S E I_{i}=-\sum_{j=1}^{n} \% V A_{i j} \cdot \ln \left(\% V A_{i j}\right)$

$\mathrm{n}_{\mathrm{i}}=$ número de actividades competitivas en la localidad $\mathrm{i}$

$\mathrm{N}_{\mathrm{i}}=$ número total de actividades en la localidad $\mathrm{i}$

$\% V A_{i j}=$ participación de VA de la actividad competitiva $\mathrm{j}$ en la localidad i

$\mathrm{i} \quad=$ indicador de localidad

El $S E I^{*}$ se obtiene a partir del índice original de Shannon $\left(S E I_{\mathrm{i}}\right)$ mediante la división de su valor entre el resultado de la máxima diversificación competitiva posible, es decir, cuando todas las actividades competitivas alcanzan un mismo peso (el logaritmo del número de actividades competitivas en cada localidad). El resultado indica la diversificación de las actividades competitivas, pero no toma en cuenta la participación de éstas en el número total de actividades. Para incorporar este efecto, se multiplicó el cociente anterior por la proporción de actividades competitivas de cada ZM y municipio $\left(n_{\mathrm{i}} / N_{\mathrm{i}}\right)$, obteniendo el índice de diversificación de actividades competitivas: $S E I^{*}$, antes llamado índice de entropía ajustado. Éste incorpora tanto el nivel de diversificación/ 
concentración como la importancia en número de dichas actividades $\mathrm{y}$, por lo tanto, es una medida más precisa de competitividad sistémica.

El rango de valores posibles del $S E I^{*}$ está entre 0 y 100 , indicando estos límites las situaciones de absoluta concentración y absoluta diversificación de las actividades competitivas, respectivamente. Se prefiere este índice sobre otros usuales como el Gini o el HHI por el efecto de compactación que le confiere el uso de logaritmos, es decir, hace menos sensible el cálculo a los valores extremos equilibrando su peso relativo ${ }^{16}$.

El cuadro 6 resume los resultados de la estimación del SEI*. Como puede observarse, el índice de entropía ajustado a pesar de presentar una gran dispersión, coincide mayormente en el ordenamiento de los grupos identificados mediante el cluster analysis. Las dos excepciones a notar refieren a Guanajuato, que mejora su posición con el $S E I^{*}$, y San José Iturbide que cae entre los de menor índice de $S E I^{* 17}$. Otras observaciones más puntuales se ofrecen en el siguiente apartado.

\subsection{Análisis de resultados}

Los resultados se refieren a los dos aspectos que hemos venido desarrollando, es decir, la importancia de las actividades competitivas por localidad y el ordenamiento de las localidades a través del índice de entropía ajustado. Ambos criterios permiten definir tres grupos de localidades a los que referiremos los resultados más puntuales.

a) El grupo o cluster 1, conformado por la media docena de ZM más importantes con más de 20 actividades competitivas cada una, también alcanza al menos $48 \%$ del VA y $41 \%$ del L con esas actividades en cada localidad. Aún entre ellos hay grandes diferencias, destacando sobre los demás Querétaro. En orden de mayor a menor, en ese grupo sobresalen

16 Es común el uso de los índices de Herfindahl-Hirschman (HHI) y de Gini para estimar situaciones de diversificación/concentración. Sin embargo, estos índices no se cuidan de ajustar los valores extremos de las medidas. Por ejemplo, si se implementara el HHI, daría mayor peso relativo a las actividades con mayor \%VA, afectando a las ZM o municipios en los que una sola actividad concentra la mayor parte del VA competitivo. Por otra parte, el índice de Gini absoluto no es indicado para un número limitado de observaciones. En otras palabras, no cumple con ciertas características básicas para ser una medida adecuada de especialización (ver Palan, 2010). Por lo tanto, se eligió el SEI* para medir la diversificación en el Bajío dada la variedad de situaciones competitivas en cada localidad.

17 San José Iturbide es un caso particular de 2 actividades competitivas de alta importancia, y las demás muy marginales. De ahí que el SEI* lo sitúa con muy baja competitividad sistémica. 
Cuadro 6

ÍNDICE DE ENTROPÍA AJUSTADO

\begin{tabular}{|c|c|c|c|c|}
\hline $\begin{array}{l}\text { ZONA METROPOLITANA O } \\
\text { MUNICIPIO IMPORTANTE }\end{array}$ & PROPORCIÓN ACTS COMPa & $\mathrm{SEI}^{\mathrm{b}}$ & $\mathrm{SEi}^{* \mathrm{c}}$ & CLUSTER \%VA-CR1 Y \% $L^{d}$ \\
\hline Querétaro (4) & 0.65 & 3.66 & 60.1 & 1 \\
\hline Guadalajara (8) & 0.48 & 3.39 & 44.3 & 1 \\
\hline San Luis Potosí (2) & 0.37 & 3.20 & 35.3 & 1 \\
\hline Aguascalientes (3) & 0.39 & 2.82 & 32.7 & 1 \\
\hline León (2) & 0.36 & 2.72 & 29.3 & 1 \\
\hline Celaya (3) & 0.27 & 2.69 & 23.9 & 1 \\
\hline San Juan del Río & 0.30 & 2.31 & 23.0 & 2 \\
\hline Irapuato & 0.30 & 2.00 & 19.4 & 2 \\
\hline Ciudad Valles & 0.22 & 2.13 & 18.7 & 2 \\
\hline Zacatecas (3) & 0.15 & 2.20 & 14.5 & 2 \\
\hline Guanajuato & 0.24 & 1.39 & 13.1 & 3 \\
\hline Salamanca & 0.28 & 1.28 & 12.8 & 2 \\
\hline Lagos De Moreno & 0.20 & 1.52 & 12.0 & 2 \\
\hline La Piedad-Pénjamo (2) & 0.13 & 1.89 & 11.5 & 3 \\
\hline Calera & 0.15 & 1.50 & 11.2 & 3 \\
\hline Región Tequilera & 0.10 & 1.60 & 8.5 & 3 \\
\hline San José Iturbide & 0.17 & 1.03 & 7.9 & 2 \\
\hline Fresnillo & 0.20 & 0.75 & 6.1 & 3 \\
\hline Ocotlán (2) & 0.15 & 0.77 & 5.2 & 3 \\
\hline Sn Fco del Rincón (2) & 0.07 & 1.07 & 5.1 & 3 \\
\hline Apaseo el Grande & 0.11 & 0.68 & 4.6 & 3 \\
\hline
\end{tabular}

Notas: a) La proporción de actividades competitivas $\left(n_{i} / N_{i}\right)$ es el cociente del número de actividades competitivas entre el número total de actividades. b) El índice Shannon de entropía se define como $S E I_{i}=-\sum_{j=1}^{n} \% V A_{i j} \cdot \ln \left(\% V A_{i j}\right)$ c) El índice de entropía ajustado se especifica como $S E I^{*}{ }_{i}=\frac{S E I_{i}}{\ln \left(n_{i}\right)} \cdot \frac{n_{i}}{N_{i}} \cdot 100$ d) Resultados del cluster analysis previamente presentados en el cuadro 5.

Fuente: Elaborado con datos de los Censos Económicos 2009 (INEGI, 2009).

Querétaro (51 actividades; alrededor de $80 \%$ en VA y L), Guadalajara (38, menos de la mitad de actividades; alrededor de $60 \%$ en VA y L), Aguascalientes (30; 75\% en VA y 49.4\% en L), San Luis Potosí (29; cerca de $50 \%$ en VA y L), León ( $27 ; 70 \%$ en VA y $62.6 \%$ en L) y Celaya (20; con $61 \%$ del VA y sólo $41.5 \%$ de L).

El orden dado por el SEI* (columna 3, cuadro 6) se corresponde con el resultado del cluster analysis de la importancia neta y el empleo competitivo del cuadro 5. Las seis ZM más importantes en VA competitivo (menos CR1) y L competitivo del grupo 1 presentan índices $S E I^{*}$ altos, aunque con mayor dispersión (23.9 a 60.1\%). Esto se ve influen- 
ciado en gran medida por la proporción de actividades competitivas que va desde 0.27 en el caso de Celaya hasta 0.65 para Querétaro. En concordancia con los resultados anteriores, Querétaro se mantiene como la ZM más diversificada en actividades competitivas. Le siguen Guadalajara y San Luis Potosí, que a pesar de ser diversificadas, cuentan con una proporción menor de actividades competitivas en comparación con la primera. Finalmente, Aguascalientes, León y Celaya presentan índices altos pero ligeramente menores a los de las tres primeras ZM; esto se podría explicar por la presencia de una o dos actividades competitivas con muy alta importancia relativa en cada una de ellas (autos en Aguascalientes, autos y cuero-piel en León, y jabones y electrodomésticos en Celaya; ver anexo 2).

b) El segundo grupo, con indicadores de desempeño competitivo más moderados, se compone de los tres municipios de mayor importancia después de los anteriores y también de otros cuatro menores. En todos ellos hay elementos de dominancia clara de algunas muy pocas actividades que no permiten hacer un mismo juicio para el municipio como un todo. Entre los criterios a resaltar es que tienen muy pocas actividades en condición competitiva. Así tenemos a los primeros que incluyen Irapuato (21 actividades competitivas; con $68 \%$ y $43 \%$ en VA y L respectivamente), San Juan del Río (20; con 56.6\% del VA y 29.6\% de L) y Salamanca (17; con $88.9 \%$ del VA y sólo $32.2 \%$ de L). En este último destaca sobremanera el efecto de derivados del petróleo (refinería de Pemex) que por sí sola aporta 60\% del VA municipal, y sumadas la química y servicios relacionados con transporte llegarían a representar $76.7 \%$ del VA y sólo $16 \%$ de L. En similar situación están los otros municipios. En Irapuato destacan derivados lácteos con 34.1\% del VA, con la suma de éste y otras tres actividades importantes (pan y tortillas, comercio de materias primas al mayoreo y, comercio de bebidas y tabaco al mayoreo) subiendo a $48.9 \%$ del VA. Estos dos casos, Salamanca e Irapuato, son representativos también de la influencia de una empresa en una actividad comerciable relativamente competitiva que se rodea de otras actividades no comerciables que también alcanzan condiciones competitivas.

El caso de San Juan del Río tal vez es un tanto diferente. Indudablemente le alcanza la influencia de la ZM de Querétaro, pues no podría entenderse la importancia que toman industrias relacionadas o desplazadas desde aquél como la industria del papel, equipo de cómputo, 
molienda de granos, aceites y grasas y servicios relacionados con el transporte. Las demás actividades se ven limitadas de crecer precisamente por la cercanía con Querétaro por lo que no ha logrado desarrollar sus propias actividades de comercio y servicios de importancia. Si se pensaran como zonas económicas, tal vez sería lógico sumar San Juan del Río a la ZM Querétaro.

Los otros municipios de menor tamaño en este grupo registran muy poca actividad competitiva una vez que deducimos la influencia de la actividad principal. Son monoproductores clásicos y todo indica que no han dado lugar a la creación de derramas de significancia. Son casos de convivencia amigable en estructuras económicas dispares. Es el caso de San José Iturbide (jabones y limpiadores con 49.7\% del VA, en compañía de productos de minerales no metálicos con 19.2\%), Lagos de Moreno (leche en polvo y evaporada con $29.0 \%$ ) y en menor medida pero también con impactos de competitividad muy limitados aplicaría a los números de Zacatecas y Ciudad Valles. Estos municipios presentan por lo general una imagen de dualidad competitiva en que coexisten pocas o hasta una sola actividad con competitividad exitosa, generalmente asociadas a una gran empresa de talla transnacional; al lado de muchas actividades tradicionales y marginales que no se ven apuradas ni necesitadas de responder a las presiones de la modernidad ni de la productividad.

Para el grupo 2 también coinciden los resultados del orden del índice $S E I^{*}$ y la agrupación obtenida del cluster analysis, con una sola excepción: San José Iturbide, el cual presenta una muy baja diversificación competitiva, como señalamos antes. Excluyendo este caso, las ZM y municipios de este grupo se caracterizan por índices $\left(S E I^{*}\right)$ menos dispersos que los del grupo 1, de 12.0 a $23.0 \%$. Este resultado está determinado en gran medida por una proporción modesta de actividades competitivas promedio en todas las localidades.

c) El tercer grupo es claramente un conjunto de muy escasa competitividad, y más aún al quitar la actividad principal. Son una versión extrema de los del segundo grupo antes descrito. Se trata de 8 municipios, entre ellos tres monoproductores de una sola actividad en una sola empresa: Apaseo el Grande, Fresnillo y Ocotlán quedan con VA competitivo por debajo de $18 \%$ una vez que se quita el porcentaje de la actividad principal. Dicha actividad da cuenta de más de la mitad del VA total en cada caso. Las actividades competitivas se limitan a 5, 12 y 
9 respectivamente, en su gran mayoría comercios y servicios no comerciables. Las otras cinco localidades Guanajuato, Calera, Región Tequilera, San Francisco del Rincón y La Piedad-Pénjamo se mantienen en poca actividad. En Guanajuato las actividades competitivas son predominantemente no comerciables y la principal es telecomunicaciones. Las demás localidades ni siquiera cuentan con que su actividad principal sea competitiva, subsisten tal cual ${ }^{18 .}$

Por último, el grupo 3 se caracteriza por contar con los índices de diversificación más bajos y, a la vez, la dispersión entre ellos es menor en comparación con los grupos 1 y 2 . Este conjunto de localidades es muy heterogéneo. Por un lado se encuentran las ZM de La Piedad-Pénjamo y San Francisco del Rincón que cuentan con un número muy reducido de actividades competitivas, relativamente diversificadas, pero que en conjunto aportan muy poco VA en comparación con el grupo 2 (ver anexo 2). Por otro lado, los municipios de Fresnillo y Apaseo el Grande y la ZM Ocotlán se caracterizan por ser monoproductores, lo cual se evidencia con índices de diversificación bajos, como ya habíamos anticipado. Las localidades de Calera y la Región Tequilera también pueden ser consideradas monoproductoras, aunque esta condición no se ve reflejada en el índice dado que la actividad más importante en cada caso no es competitiva. Finalmente, Guanajuato, quien tampoco cuenta con su actividad principal competitiva, representa un caso aislado dentro de este grupo. Su índice de diversificación lo sitúa en el grupo 2 pero por una diferencia mínima.

\section{CONCLUSIONES}

En este trabajo hemos intentado llegar a apreciaciones generales de la región definida como Bajío pero sin perder de vista las especificidades que caracterizan a las diferentes localidades. La apreciación más general es que el Bajío como conjunto padece de poca competitividad,

18 Debemos hacer advertencia de algunos resultados asociados a la limitación de acceso a datos. Es el caso para el municipio de Calera y su actividad principal, la elaboración de cerveza (Cervecería Grupo Modelo) que no reporta salarios en sus cuentas de ese municipio. Otro caso es la Región Tequilera para la cual no incluimos al municipio de Zapotlanejo, dada su cercanía con la ZM Guadalajara, pero que aporta el $15 \%$ de la producción nacional de bebidas destiladas de agave. Para la Piedad-Pénjamo las actividades agropecuarias son de mayor importancia, como se mostró en otro trabajo (Unger, et al. 2014). 
tanto por productividad laboral como por nivel de salarios en la comparación con la media nacional de sus actividades principales.

Este resultado se obtiene al estimar la competitividad de las ZM y los municipios del Bajío mediante un índice relativamente simple que recoge tanto la productividad laboral relativa a la nacional, como la derrama de las ganancias por productividad reflejada en el uso de capital humano con mejores salarios. El mejor resultado esperado sería tener ventajas sobre las medias nacionales en ambos indicadores. No obstante, ese resultado se presenta en pocos casos, como destacaremos enseguida.

Las estimaciones para las localidades que componen el Bajío muestran una extensa variedad de condiciones competitivas. En suma resulta un Bajío sumamente heterogéneo en cuanto a competitividad. Esto mismo impide quedarnos en las generalizaciones, pues encontramos situaciones que obligan a reconocer y destacar el papel individual de las actividades que alcanzan buena competitividad en cada localidad. También logramos precisar una variedad de diferentes motivos por los que se alcanza dicha condición, algunos muy particulares del sector y la empresa líder, otros de más amplia interacción de influencias entre las actividades de la localidad.

Los resultados más puntuales comienzan por distinguir a las tres localidades en que la media aritmética de todas las actividades alcanza condición competitiva: Querétaro $\left(\mathrm{C}^{* *}=1.17\right)$, Guadalajara $\left(\mathrm{C}^{* *}=1.01\right)$ e Irapuato $\left(\mathrm{C}^{* *}=1.00\right)$. Los demás quedan por debajo de la media nacional. Aún entre estos tres relativamente competitivos hay diferencias, pues únicamente Querétaro mantiene el nivel de ventajas tanto en productividad como en las salariales, y lo logra también en actividades comerciables por igual que en las no comerciables. Guadalajara e Irapuato, por su parte, solamente alcanzan competitividad en las actividades no comerciables. En particular, Irapuato descansa muy significativamente en la influencia de una actividad que impacta el cálculo de su media aritmética, y no puede afirmarse su condición competitiva de conjunto. Más grave resulta que las otras 18 de las ZM y municipios del Bajío caen muy por debajo en ambos indicadores, lo cual se refleja en una media de competitividad aproximadamente a la mitad de la del país. Este mismo patrón se repite con muy pocas variantes al distinguir entre actividades comerciables y no comerciables. 
El resultado promedio se complementa con la apreciación del grado de alcance de la competitividad sistémica o de conjunto. Este criterio atribuye mejores ventajas competitivas a una localidad cuando las competitividades son compartidas entre un número más grande de actividades en el espacio cercano de la localidad, sean estas ZM o los municipios individuales. El ejercicio identificó tres grupos o clusters de localidades en cuanto a la importancia económica de las actividades competitivas (importancia neta al exceptuar la principal) y la importancia que alcanza el empleo en esas mismas actividades competitivas. Las particularidades de cada grupo son dignas de subrayarse pues nos permiten concluir que no puede asumirse la extrapolación o expectativa de desarrollar las regiones por contagio entre vecinos.

El grupo de pertenencia al cluster 1 es significativamente más competitivo que los otros dos en términos de competitividad sistémica. Es representativo de la media docena de ciudades o ZM que han llegado a reunir condiciones competitivas relativamente favorables y compartidas. Cuentan con más de 20 actividades competitivas cada una, y alcanzan a representar alrededor de la mitad de la actividad económica y del empleo con esas actividades. Aún entre ellos hay grandes diferencias, destacando de nueva cuenta sobre los demás Querétaro, que por sí solo pertenece al selecto grupo de entidades líderes nacionalmente. Le siguen en orden de competitividad sistémica aunque en otro nivel las ZM de Guadalajara, Aguascalientes, San Luis Potosí, León y Celaya.

El segundo grupo, el cluster 2, muestra indicadores de desempeño competitivo aún más moderados. Está compuesto de los tres municipios de mayor importancia después de los anteriores y de otros cuatro menores. Los primeros incluyen Irapuato, San Juan del Río y Salamanca. Este último e Irapuato, son representativos también de la influencia de una empresa en una actividad comerciable relativamente competitiva que se rodea de otras actividades no comerciables, algunas de las cuales también pueden alcanzar condiciones competitivas. En cuanto a San Juan del Río, quizás podría considerarse aportando capacidades específicas complementarias a la ZM Querétaro, a la vez que aprovecha las amenidades terciarias de esa zona económica. La idea de una competitividad sistémica de alcances muy modestos aplica por igual para Salamanca, San José Iturbide, Irapuato, Apaseo el Grande, Ocotlán y Fresnillo, al limitar la consideración a la importancia neta de las competitivas sin incluir el impacto de la actividad principal. 
Los otros municipios que integran el Bajío son de menor tamaño y registran muy poca actividad competitiva, sobretodo una vez que deducimos la influencia de la actividad principal. Se caracterizan como monoproductores clásicos y todo indica que no han dado lugar a la creación de derramas de significancia. Coexisten una sola o muy pocas actividades con competitividad exitosa, generalmente asociadas a una gran empresa de talla transnacional; al lado de muchas actividades tradicionales, marginales y atrasadas. En algunos casos, como serían Apaseo el Grande, Fresnillo y Ocotlán quedan limitados a un VA competitivo por debajo del $20 \%$ una vez que se quita el porcentaje de la actividad principal. Dicha actividad suele dar cuenta de más de la mitad del VA total en cada caso. Otras cinco localidades son Guanajuato, Calera, Región Tequilera, San Francisco del Rincón y La PiedadPénjamo que se mantienen con poca actividad y en condiciones mucho menos promisorias.

Las implicaciones de política deben referirse a las condiciones señaladas para cada grupo de localidades. El tipo de política o los matices de políticas públicas tendrán que ajustarse a las mismas tres situaciones que han quedado en evidencia: las localidades poco competitivas y sin derramas de competitividad sistémica tendrán que ser objeto de acciones más amplias, incluyendo la promoción de infraestructura de todo tipo, en tanto las otras pueden merecer acciones más puntuales. En estos casos, también vale distinguir las acciones de acuerdo con la vocación revelada; tal vez es el caso de la mayoría de las ZM, excluyendo Querétaro, las cuáles están más orientadas al comercio y servicios de naturaleza no comerciables. En particular para las ZM de León y Guadalajara se requiere de una política industrial más activa que les devuelva competitividad en sectores industriales comerciables. 


\section{Anexo 1. DELIMITACIÓN DEL BAJÍO: 209 MUNICIPIOS}

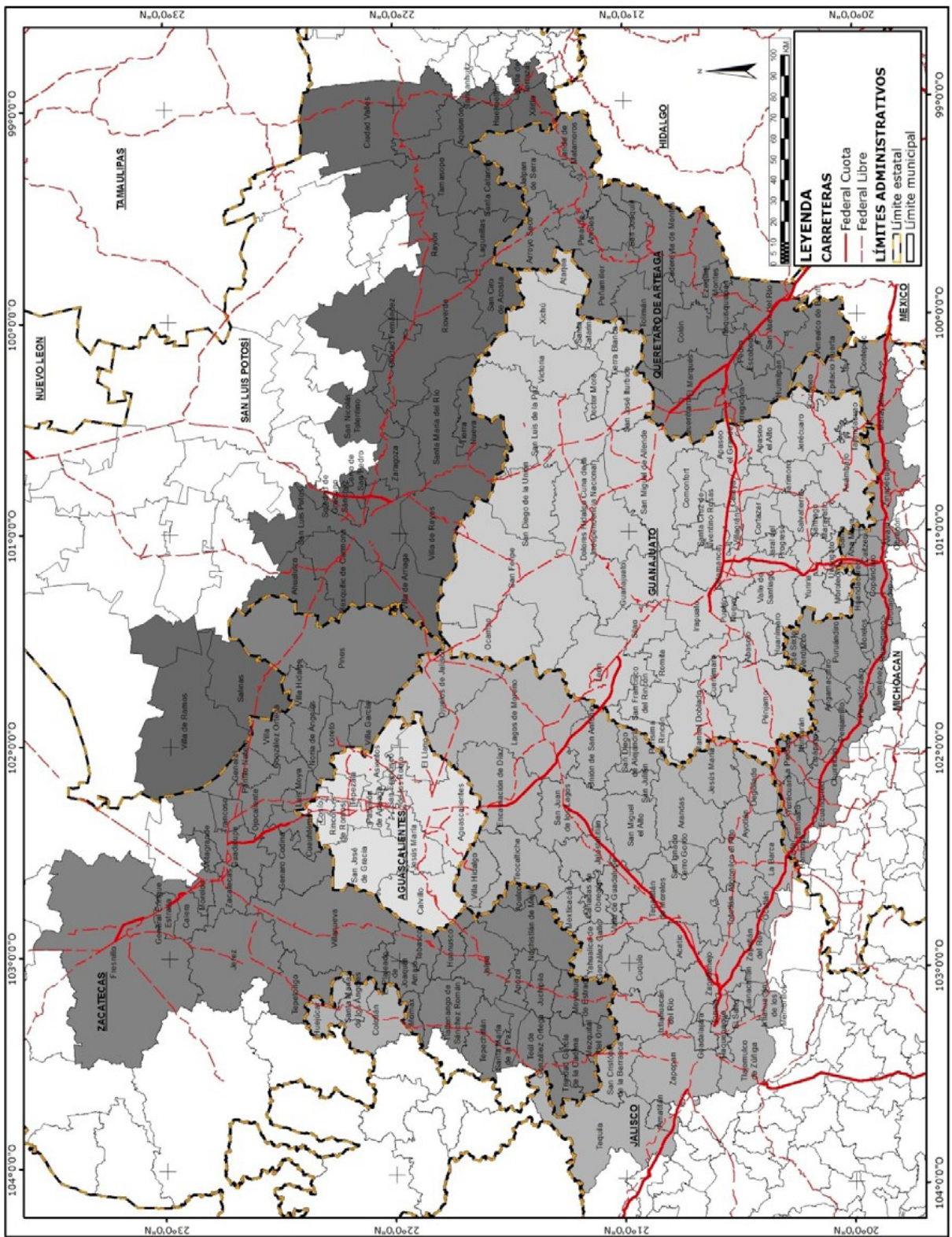

Fuente: Unger et al. (2014a). 
ANEXO 2. ACTIVIDADES PRINCIPALES (VA > 1.2\%), 2009

\begin{tabular}{|c|c|c|c|c|c|c|c|c|}
\hline \multirow[b]{2}{*}{$\begin{array}{l}\mathrm{C} / \\
\mathrm{NC}\end{array}$} & \multirow[b]{2}{*}{82 ACTIVIDADES } & \multicolumn{5}{|c|}{ BAJIO } & \multicolumn{2}{|c|}{ NACIONAL } \\
\hline & & $\begin{array}{l}\text { VA } \\
\text { miles de } \\
\text { millones }\end{array}$ & $\begin{array}{l}\text { W miles } \\
\text { de } \\
\text { millones }\end{array}$ & $\begin{array}{l}\mathrm{L} \text { miles de } \\
\text { personas }\end{array}$ & $\% \mathrm{VA}$ & $\begin{array}{l}\text { \%L 82/ } \\
\text { TOT }\end{array}$ & $\% \mathrm{VA}$ & $\begin{array}{c}\% \mathrm{~L} 82 / \\
\text { TOT }\end{array}$ \\
\hline NC & 2211 ENERGÍA ELÉCTRICA & 22,165 & 5,456 & 17.7 & 3.90 & 0.61 & 4.30 & 0.62 \\
\hline C & 3361 AUTOMÓVILES Y CAMIONES & 19,105 & 1,905 & 8.5 & 3.37 & 0.29 & 1.64 & 0.25 \\
\hline NC & $\begin{array}{c}5171 \text { TELECOMUNICACIONES } \\
\text { ALÁMBRICAS }\end{array}$ & 16,936 & 5,080 & 18.7 & 2.98 & 0.64 & 1.82 & 0.54 \\
\hline NC & 434 MAT PRIMAS (MAY) & 16,676 & 3,812 & 64.4 & 2.94 & 2.22 & 1.80 & 2.02 \\
\hline $\mathrm{C}$ & 332 PRODUCTOS METÁLICOS & 11,786 & 3,643 & 65.9 & 2.08 & 2.27 & 1.31 & 1.83 \\
\hline NC & 4311 ABARROTES Y ALIMENTOS (MAY) & 11,759 & 3,089 & 43.3 & 2.07 & 1.50 & 1.30 & 1.36 \\
\hline NC & 2221 SUMINISTRO DE AGUA & 11,260 & 1,489 & 10.8 & 1.98 & 0.37 & 0.91 & 0.55 \\
\hline NC & $\begin{array}{l}\text { 4611, } 4612 \text { ABARROTES, ALIMENTOS/ } \\
\text { BEBIDAS (MEN) }\end{array}$ & 10,758 & 1,966 & 234.0 & 1.89 & 8.07 & 1.45 & 9.58 \\
\hline NC & $\begin{array}{l}541 \text { SERV PROFESIONALES, } \\
\text { CIENTÍFICOS Y TÉCNICOS }\end{array}$ & 10,434 & 4,360 & 79.3 & 1.84 & 2.73 & 1.88 & 2.84 \\
\hline NC & 5613 SERVICIOS DE EMPLEO & 10,368 & 8,869 & 88.6 & 1.83 & 3.06 & 1.82 & 3.57 \\
\hline C & $\begin{array}{l}316 \text { CURTIDO CUERO Y PIEL, } \\
\text { PRODUCTOS DE CUERO }\end{array}$ & 10,310 & 4,897 & 102.7 & 1.82 & 3.54 & 0.26 & 0.70 \\
\hline C & $\begin{array}{c}3241 \text { DERIVADOS DEL PETRÓLEO Y } \\
\text { CARBÓN }\end{array}$ & 10,287 & 1,868 & 6.0 & 1.81 & 0.21 & 0.46 & 0.16 \\
\hline C & $\begin{array}{c}3112 \text { MOLIENDA GRANOS, SEMILLAS, } \\
\text { ACEITES, GRASAS }\end{array}$ & 9,742 & 1,179 & 9.7 & 1.72 & 0.34 & 0.54 & 0.17 \\
\hline C & 325 QUÍMICA & 9,579 & 1,950 & 16.7 & 1.69 & 0.58 & 3.08 & 0.53 \\
\hline C & $\begin{array}{c}3256 \text { JABONES, LIMPIADORES Y PREP } \\
\text { TOCADOR }\end{array}$ & 9,566 & 1,191 & 9.1 & 1.68 & 0.31 & 0.64 & 0.23 \\
\hline NC & $\begin{array}{c}722 \text { SERVICIOS DE ALIMENTOS Y } \\
\text { BEBIDAS }\end{array}$ & 9,423 & 2,913 & 181.1 & 1.66 & 6.25 & 1.47 & 7.04 \\
\hline C & $\begin{array}{l}435 \mathrm{MAQ} \text {, EQUIPO Y MOBILIARIO } \\
\text { (MAY) }\end{array}$ & 9,251 & 1,962 & 30.7 & 1.63 & 1.06 & 0.97 & 0.82 \\
\hline NC & 3118 PAN Y TORTILLAS & 9,031 & 1,998 & 46.1 & 1.59 & 1.59 & 1.00 & 2.09 \\
\hline NC & 3261 PLÁSTICO & 8,644 & 2,502 & 41.6 & 1.52 & 1.43 & 0.93 & 0.97 \\
\hline C & $\begin{array}{c}312111 \text { REFRESCOS Y OTRAS NO } \\
\text { ALCOHÓLICAS }\end{array}$ & 8,543 & 2,630 & 19.4 & 1.50 & 0.67 & 0.89 & 0.32 \\
\hline C & 3122 TABACO & 8,220 & 151 & 0.6 & 1.45 & 0.02 & 0.54 & 0.02 \\
\hline NC & $\begin{array}{l}561 \text { SERVICIOS DE APOYO A LOS } \\
\text { NEGOCIOS }\end{array}$ & 8,253 & 4,289 & 91.4 & 1.45 & 3.15 & 3.29 & 6.69 \\
\hline NC & 336390 OTRAS AUTOPARTES & 8,194 & 1,703 & 17.0 & 1.44 & 0.59 & 0.65 & 0.45 \\
\hline C & $\begin{array}{c}311513 \text { DERIVADOS Y FERMENTOS } \\
\text { LÁCTEOS }\end{array}$ & 7,709 & 351 & 5.3 & 1.36 & 0.18 & 0.30 & 0.12 \\
\hline C & $\begin{array}{c}312142 \text { BEBIDAS DESTILADAS DE } \\
\text { AGAVE }\end{array}$ & 7,518 & 521 & 5.5 & 1.32 & 0.19 & 0.18 & 0.04 \\
\hline C & 312120 ELABORACIÓN DE CERVEZA & 7,463 & 351 & 4.5 & 1.31 & 0.16 & 0.46 & 0.07 \\
\hline C & 3254 FARMACÉUTICOS & 7,140 & 2,376 & 17.8 & 1.26 & 0.61 & 1.39 & 0.40 \\
\hline NC & $\begin{array}{c}522 \text { INTERMED CREDITICIA Y FINAN } \\
\text { NO BURSÁTIL }\end{array}$ & 7,145 & 1,891 & 17.4 & 1.26 & 0.60 & 6.61 & 1.72 \\
\hline C & 21222 MINERÍA ORO Y PLATA & 6,683 & 586 & 3.8 & 1.18 & 0.13 & 0.32 & 0.07 \\
\hline $\mathrm{C}$ & 322 IND PAPEL & 6,654 & 1,767 & 18.9 & 1.17 & 0.65 & 0.72 & 0.50 \\
\hline
\end{tabular}

Fuente: INEGI (2009) 


\section{ANEXO 3. ZM Y MUNICIPIOS IMPORTANTES DEL BAJÍO (IDENTIFICADOS POR CÓDIGO DEL INEGI)}

\begin{tabular}{|c|c|}
\hline Guadalajara (8) & Región Tequilera (5) \\
\hline 14039 Guadalajara & 14093 Tepatitlán De Morelos \\
\hline 14120 Zapopan & 14008 Arandas \\
\hline 14098 Tlaquepaque & 14013 Atotonilco El Alto \\
\hline 14070 El Salto & 14094 Tequila \\
\hline 14097 Tlajomulco De Zúñiga & 14005 Amatitán \\
\hline 14101 Tonalá & Zacatecas (3) \\
\hline 14044 Ixtlahuacán De Los Membrillos & 32017 Guadalupe \\
\hline 14051 Juanacatlán & 32056 Zacatecas \\
\hline Querétaro (4) & 32032 Morelos \\
\hline 22014 Querétaro & Ocotlán (2) \\
\hline 22011 El Marqués & 14063 Ocotlán \\
\hline 22006 Corregidora & 14066 Poncitlán \\
\hline 22008 Huimilpan & San Francisco Del Rincón (2) \\
\hline León (2) & 11031 San Francisco Del Rincón \\
\hline 11020 León & 11025 Purísima Del Rincón \\
\hline 11037 Silao & La Piedad-Pénjamo (2) \\
\hline San Luis Potosí (2) & 16069 La Piedad \\
\hline 24028 San Luis Potosí & 11023 Pénjamo \\
\hline 24035 Soledad De Graciano Sánchez & 11017 Irapuato \\
\hline Aguascalientes (3) & 11017 Irapuato \\
\hline 01001 Aguascalientes & 22016 San Juan Del Río \\
\hline 01005 Jesús María & 11027 Salamanca \\
\hline 01011 San Francisco De Los Romo & 11015 Guanajuato \\
\hline Celaya (3) & 32010 Fresnillo \\
\hline 11007 Celaya & 32005 Calera \\
\hline 11044 Villagrán & 11005 Apaseo el Grande \\
\hline \multirow[t]{2}{*}{11009 Comonfort } & 14053 Lagos De Moreno \\
\hline & 11032 San José Iturbide \\
\hline
\end{tabular}

Fuente: Inegi (2009).

\section{BiBLIOGRAFÍA}

Abdel, Guillermo (2000). "Regional and Local System of Innovation in Aguascalientes", en M. Cimoli (ed.). Developing Innovation Systems: México in a Global Context, Nueva York: Continuum.

Balassa, Bela (1965). Trade liberalisation and revealed comparative advantage, Review of Manchester School Economics and Social Sciences, pp. 99-123. Baldwin, R; R. Forslid; P. Martin; G. Ottaviano y F. Robert-Nicoud (2003). Economic Geography and Public Policy. New Jersey: Princeton University Press.

Cabrero, Enrique (coordinador) (2009). Competitividad de las ciudades en México: la nueva agenda urbana. México: CIDE. 
Conapo (2000), Indicadores sobre migración a Estados Unidos, índice y grado de intensidad migratoria por municipio, 2000, en: http://www.conapo.gob. $\mathrm{mx} /$

Corral, Carlos (2000). México 2020 un Enfoque Territorial del Desarrollo; Vertiente Urbana. Síntesis Ejecutiva. Secretaría de Desarrollo Social, Colegio de Arquitectos de la Ciudad de México y el Instituto de Investigaciones Económicas de la UNAM, México.

Diewert, W. Erwin y Alice O. Nakamura (2007). "The Measurement of Productivity for Nations", J. J. Heckman y E.E. Leamer (eds.), Handbook of Econometrics, vol. 6, chapter 66, Elsevier.

EGAP (2010). La Competitividad de los Estados Mexicanos 2010, fortalezas ante la crisis. ITESM.

Estrada, Salvador (2006). "Diferencias regionales en la conducta tecnológica de las empresas manufactureras mexicanas: el caso de Guanajuato", Economía, Sociedad y Territorio, Colegio Mexiquense, pp.821-869.

Fujita, M.; P. Krugman. y A. J. Venables (1999). The Spatial Economy. Cambridge, MA: The MIT Press.

Garduño, Rafael; José Eduardo Ibarra y Rafael Dávila (2013). La medición de la competitividad en México: ventajas y desventajas de los indicadores. Documento de Trabajo E-557, CIDE.

Huggins, R. y Hiro Izushi (2008). UK Competitiveness Index 2008. University of Wales Institute, Cardiff - UWIC: Centre for International Competitiveness - Cardiff School of Management

IMCO (2012). Índice de Competitividad Urbana 2012. México, D.F.

INE (2006). Atlas de la Cuenca Lerma-Chapala: Construyendo una visión conjunta. México: INE

INEGI (2009). Censos Económicos 2009. Sistema Clasificación Industrial de América del Norte (SCIAN), Sistema Automatizado de Información Censal SAIC 5.

INEGI (2011). Directorio Estadístico Nacional de Unidades Económicas (DENUE 03/2011). Estados Unidos Mexicanos.

INEGI (2012). Delimitación de las Zonas Metropolitanas de México 2010. México.

Krugman, Paul (1991). "Increasing Returns and Economic Geography", The Journal of Political Economy, 99 (9), 483-499

Krugman, Paul (1996). Development, Geography and Economic Theory, MIT Press.

Meyer, John (1963). "Regional Economics: A Survey", The American Economic Review, 53 (1), Part 1, American Economic Association, pp. 19-54

OECD (2007). Competitive Regional Clusters: National Policy Approaches.

OECD (2009). Reviews of Regional Innovation: 15 Mexican States

Palan, Nicole (2010). Measurement of Specialization, The Choice of Indices. FIW Working Paper, $\mathrm{N}^{\circ}$ 62, December.

Porter, Michael (1990). The Competitive Advantage of Nations, Londres: MacMillan. 
Porter, Michael(1992). Competitive Advantage: Creating and Sustaining Superior Performance. PA Consulting Group.

Rodríguez-Posé, Andrés (2000). Local Production Systems and Economic Performance in France, Germany, Italy, and the United Kingdom. Mimeo presentado en el Seminario Internacional NAFIN/UNAM Aprendiendo de las regiones en el mundo: ¿cómo combatir la desigualdad productiva?, mayo 2000, México.

Rodrik, Dani (2007). One economics, many recipes: globalization, institutions, and economic growth. Princeton University Press.

Schwab, K. y Michael Porter (2007). The Global Competitiveness Report 2007-2008. World Economic Forum. Geneva, Switzerland.

Sedesol, Conapo, Inegi (2004). Delimitación de las Zonas Metropolitanas. México: Sedesol, Conapo, Inegi.

Sobrino, Jaime (2003), Zonas Metropolitanas de México en 2000: Conformación territorial y movilidad de la población ocupada", Estudios Demográficos y Urbanos, El Colegio de México, septiembre-diciembre, Núm. 054, México, pp. 461-507.

Turok, Ivan (2004). "Cities, Regions and Competitiveness", Regional Studies, 38 (9), pp. 1069-1083.

Unger, Kurt (2009). “Apertura y empleos: la economía de los sectores comerciables y no comerciables de las regiones de México", en J. Arroyo y S. Berumen (coords), Migración a Estados Unidos, UdeG/SEGOB/Instituto Nacional de Migración, 2009.

Unger, Kurt (2010). Globalización y clusters regionales en México: un enfoque evolutivo, Fondo de Cultura Económica, México.

Unger, Kurt (2012). Especializaciones reveladas y condiciones de competitividad en las entidades federativas de México, Documento de Trabajo E-530, CIDE.

Unger, Kurt y Luz Saldaña (1999). "Industrialización y progreso tecnológico: una comparación entre las regiones de México", Estudios Sociológicos, XVII (51), septiembre-diciembre 1999, México, pp. 633-682.

Unger, Kurt, José Eduardo Ibarra y Rafael Garduño (2014a). "Especializaciones reveladas y ventajas competitivas en el Bajío Mexicano”, Econoquantum, Vol.11, n.2, pp. 41-74.

Unger, Kurt; Diana Flores y Lizet A. Pérez (2014b), Competitividad y Especialización en el Bajío Mexicano: ¿Acaso una región homogénea?. Documento de Trabajo E-569, CIDE.

Unger, Kurt, Diana Flores y José Eduardo Ibarra (2014c), "Productividad y capital humano: Fuentes complementarias de la competitividad en los estados de México", El Trimestre Económico, No. 324, Vol. LXXXI(4), Octubre-Diciembre 2014.

Van Beveren, Ilke (2012). "Total Factor Productivity Estimation: A Practical Review", Journal of Economic Surveys, 26(1), pp. 98-128. 